\title{
Kommunikationskonflikte und Fachsprachengebrauch
}

\author{
"Und das Streben, eine reine, \\ gefühllose Erkenntnissprache \\ zu schaffen, ist ebenso begreif- \\ lich, wie es aussichtslos ist." \\ (Karl Otto Erdmann) \\ "Die Biene ist in ihrer sozial- \\ relevanten Struktur als sozial- \\ ökonomisches Phänomen zur \\ Suplementation der spätkapitali- \\ stischen Gesellschaft mit einer \\ frustrationshemmenden Substan $z$ \\ namens Honig darzustellen. Dabei \\ muß auf ihre Funktion zurRepres- \\ sion unterpriviligierter Schichten, \\ sogenannter Imker, durch gesell- \\ schaf tlich nicht erzwungene Infi- \\ zierung toxischer Substanzen und \\ die Bewältigung der dadurch ent- \\ stehenden Konflikte eingegangen \\ werden." (Vorschlag für den Biologie- \\ unterricht an Hessischen Schulen, \\ um darzustellen, daß Bienen uns \\ mit Honig versorgen und stechen \\ können)
}

\section{Drei Thesen als Vorbemerkung}

Ich möchte in diesem Beitrag ${ }^{1}$ für folgende Thesen plädieren:

(1) Die Fachsprachenforschung sollte sich, im stärkeren Maße als dies bisher geschehen ist, mit dem Fachsprachen $\mathrm{g} \mathrm{e} \mathrm{b} \mathrm{ra} \mathrm{u} \mathrm{ch}$ in typisierbaren Interaktionszusammenhängen befassen. ${ }^{2}$

(2) Ein weitgehend vernachlässigtes Forschungsgebiet ist die mündliche und schriftliche, wissenschaftliche Sprachkommunikation unter Fachwissenschaftlern. Die Fachsprachenforschung sollte sich intensiver als bisher mit der fachinternen und fächerübergreifenden sprachlichen Kommunikation unter Wissenschaftlern befassen ${ }^{3}$. 
(3) Ich gehe a posteriori davon aus, daß nicht nur die sprachliche Kommunikation zwischen Fachleuten und Laien sowie die interfachliche Kommunikation, sondern auch die fachinterne, fachsprachliche Kommunikation nicht in dem Sinne zuverlässig funktioniert, daß die jeweils angestrebten Kommunikationsziele stets sicher erreicht werden können; ich setze damit voraus, daß Kommunikationskonflikte durch Fachsprachengebrauch in den verschiedenen fachspezifischen Kommunikationsbereichen oder Handlungsräumen auftreten können. Falls diese Voraussetzung richtig ist und falls man die institutionellen Bedingungen und das Funktionieren der Kommunikation im Fach studieren will, halte ich es für eine erfolgversprechende Forschungsstrategie, zunächst fachsprachlich bedingte Konflikte in einem Kommunikationsbereich zu studieren, den man aus eigener Erfahrung einigermaßen kennt. ${ }^{4}$

These (1) spricht sich für eine Pragmatisierung dafür geeigneter Fragestellungen innerhalb der Fachsprachenforschung aus. Mit These (2) wird ein Hinweis auf ein brach liegendes, aber m.E. fruchtbares Forschungsfeld gegeben. Die These (3) enthält einen allgemeinen methodologischen Vorschlag, wie man die ersten Furchen in das unbestellte Feld ziehen kann.

Insgesamt möchte ich mit meinen nachfolgenden Ausführungen lediglich versuchen, exemplarisch darauf hinzuweisen, daß die herkömmlichen Forschungsinteressen innerhalb der Fachsprachenforschung erweitert werden sollten, und zwar in Richtung auf die Erforschung des Fachsprachengebrauchs.

\section{Zwei Verstehensebenen für bestimmte Fachausdrücke?}

Die Beschäftigung mit Kommunikationskonflikten im Bereich wissenschaftlicher Kommunikation macht nachdenklich. Sollte jemand die Vorstellung vom rational und überwiegend sachlich argumentierenden Wissenschaftler haben, der möglichst ökonomisch eine weitgehend präzise, fachspezifische Zwecksprache ${ }^{5}$ diszipliniert verwendet, um Erkenntnis zu gewinnen oder anderen mitzuteilen, der wird diese bei der Beschäftigung mit Kommunikationskonflikten erheblich korrigieren müssen. Spätestens beim Nachdenken über die möglichen Ursachen und Konsequenzen von Kommunikationskonflikten treten im Gefolge weiterreichender Fragen, die ich kommunikationsethische nennen möchte, auch Fragen auf wie z.B. diese: Wie lassen sich Kommunikationskonflikte von vornherein, d.h. prinzipiell und prophylaktisch vermeiden? Durch Kritik der oder einer Wissenschaftssprache? Durch Kritik bestimmter Sprachgebräuche bestimmter Wissenschaftler oder wissenschaftlicher Schulen? 
Durch allgemeine oder spezielle sprachtherapeutische Vorschläge? Durch Diskurs?

Die Geschichte der wissenschaftlichen Sprachkritik und auch deren Kapitel "Kritik der Wissenschaftssprache und des wissenschaftlichen Sprechens und Schreibens" ist noch nicht geschrieben. Soweit ich diese Geschichte kenne, neige ich dazu, die Frage eher negativ zu beantworten; d.h.: ich sehe keine Möglichkeit für ein wissenschaftlich begründbares, allgemein anwendbares Rezeptbuch zur sicheren Vermeidung von Kommunikationskonflikten, auch nicht für den Bereich der wissenschaftlichen Kommunikation. ${ }^{6}$

Ich bin aber dennoch optimistisch genug anzunehmen, daß Kenntnisse der institutionellen, der kommunikatorinternen, der sprachbedingten und situationsspezifischen Ursachen, der unterschiedlichen Strukturvarianten, der verschiedenen Typen sowie der individuellen und gesellschaftlichen Konsequenzen von Kommunikationskonflikten dazu beitragen können, diese - im konkreten Fall ihres Auftretens - wenigstens als solche zu erkennen, so daß man sie von sozialen und Meinungskonflikten unterscheiden und intrakommunikativ beherrschen und gegebenenfalls im Nachhinein mit verbalen Mitteln in wechselseitigen hermeneutischen Verständigungsbemühungen diskutant aufklären kann derart, daß die jeweils angestrebten kommunikativen Ziele erreicht werden können. -

In den Vorbemerkungen hatte ich betont, daß ich versuchen möchte, für eine Erweiterung der Forschungsinteressen innerhalb der Fachsprachenforschung zu plädieren. Wenn man sich mit Kommunikationskonflikten beschäftigt hat, dann schätzt man einen solchen Versuch angesichts eines relativ heterogenen Großauditoriums ziemlich skeptisch ein. Er kann m.E. allenfalls dann gelingen, wenn ich mit der komplexen Sprechhandlung, mit der ich vor einigen Minuten begonnen habe, nämlich mit der Sprechhandlung nach dem Muster 'einen wissenschaftlichen Vortrag halten', nach Ende des Vortrages zumindest derart erfolgreich war, daß wenigstens einige der hier Anwesenden wenigstens den inhaltlichen Kern meiner Ausführungen akzeptiert haben. Diesen zweiten Grad des Erfolgreichseins, nämlich akzeptiert zu werden, kann man allerdings nur unter bestimmten Bedingungen überhaupt anstreben bzw. erreichen. Die wichtigste Bedingung, die erfüllt sein muß, ist die Verstehensbedingung; dies heißt: Strebt ein Vortragender an, daß seine Ausführungen akzeptiert werden, muß er zunächst dafür Sorge tragen, sprachlich verstanden zu werden. Ohne weitere Rechtfertigung und auch ohne eine Begründung, die nur aufgrund einer eingehenden Analyse des kommunikationslogischen Dilemmas gegeben werden könnte, in dem sich jeder Vortragende angesichts eines so unterschiedlich zusammengesetzten Publikums befindet, 
werde ich weitgehend auf die Verwendung sehr spezieller Fachausdrücke, die aus elaborierten theoretischen Zusammenhängen stammen und die gerade bei der Erforschung von Kommunikationskonflikten benutzt werden (!), verzichten. ${ }^{7}$ Dies bedeutet zugleich, daß sehr spezielle Sachverhalte nicht zur (Fach-) Sprache kommen werden, ein Verzicht, der mit meiner Absicht, das gegebene Thema hier zu behandeln, nicht kollidiert. Ganz ohne die Verwendung von Fachausdrücken wird es indessen dennoch nicht gehen. Denn auf die - durch den Gebrauch von Fachausdrücken keineswegs stets, aber doch häufig - erreichbare kommunikative Okonomie, die u.a. dadurch ermöglicht wird, daß der je geäußerte $F$ achausdruck vorausgesetztes Fachwissens der Zuhörer aktivierend in Anspruch nimmt, kann nicht gänzlich verzichtet werden.

Um jedoch Kommunikationskonflikte, die durch den Gebrauch von wissenschaftssprachlichen Ausdrücken entstehen können, von vornherein weitgehend selbst zu vermeiden, möchte ich auch nachfolgend so vorgehen, wie bisher bereits geschehen, und zwar folgendermaßen: Ich werde eine Reihe von Fachausdrücken, die zum Teil durch Definitionsketten 8 untereinander verbunden sind, so verwenden, daß sie auf zwei unterschiedlichen Verstehensebenen sprachlich verstanden werden können. Was ich mit der - durchaus metaphorischen Redeweise von den 'Verstehensebenen' meine, möchte ich nun an einem einfachen Beispiel erläutern, das aus fünf authentischen Textausschnitten besteht.

BEISPIEL Nr. 1

In nicht-wissenschaftlicher Rede sind folgende Satzäußerungen belegt:

(1) Die Ausfübrungen Genschers zur Terrorismusbekämpfung können von den $S P D-L i n k e n$ auf keinen Fall a $k$ z $p$ t $i$ er $t$ werden. [Hess. Rundfunk, polit. Kommentar]

(2) Der Verlauf des SPD-Parteitages bat deutlich gemacht, daß Brandt mit seinem Grundsatzreferat e $r$ folgre i $\mathrm{c} b$ war. [Hess. Rundfunk, polit. Kommentar]

(3) Ibre Darstellung des Unfallhergangs babe ich nicht in allen Einzelheiten ve rs t a $n d e n$, und muß daber Folgendes fragen. [Rechtsanwalt zum Unfallgegner]

In einem wissenschaftlichen Text findet man folgende Textausschnitte:

(4) "Der Begriff des E r f o l g r e i c h se in s bezieht sich auf die Konsequenzen von Sprechakten in der weiteren Entwicklung der Interaktionssituation. E r f o l g r e i c h s e in ist ein Prädikat für bestimmte Sprechakte. [...]

Bei den Sprechakten, die eine neue Interaktionsbedingung einführen, gehören zum vollen Erfolg drei Elemente:

1. Der Adressat erkennt (gemäß der Intention des Sprechers), daß der Sprecher eine bestimmte Einstellung ausdrückt: 
$\mathrm{I}_{\mathrm{S}} \mathrm{E}_{\mathrm{H}} \varphi_{\mathrm{S}} \rightarrow \mathrm{E}_{\mathrm{H}} \varphi_{\mathrm{S}} \mathrm{P}$

2. Der Adressat übernimmt (gemäß der Intention des Sprechers) eine korrespondierende Einstellung:

$\mathrm{I}_{\mathrm{S}} \varphi_{\mathrm{H}} \mathrm{P} \rightarrow \varphi_{\mathrm{H}} \mathrm{P}$

3. Der Adressat oder der Sprecher selbst (je nach Art des Sprechaktes) erfüllt die eingeführte Interaktionsbedingung (Obligation) :

z.B. $\mathrm{O}_{\mathrm{H} / \mathrm{S}} \mathrm{A} \rightarrow$ Erfüllt $\mathrm{O}_{\mathrm{H} / \mathrm{S}} \mathrm{A}$

Diese drei Arten (oder Grade) des Erfolgreichseins lassen sich abküzend mit den Begriffen Verstehen, A kzeptieren und $\mathrm{E}$ r füllen kennzeichnen." [Wunderlich 1976, 115 f.].

"Er [mein Versuch] kann m.E. allenfalls dann gelingen, wenn ich mit der komplexen Sprechhandlung [...] nach Ende des Vortrages derart e $r$ f o l g $r$ e i $\mathrm{ch}$ w a $r$, daß wenigstens einige der hier Anwesenden wenigstens den inhaltlichen Kern meiner Ausführungen a $k z$ e $p t$ i e $r t$ haben. Diesen zweiten Grad des $\mathrm{E} r$ f o $\mathrm{lg}$ re i c h s e in s, nämlich a $\mathrm{kz}$ e p t i e r t zu werden, kann man allerdings nur unter bestimmten Bedingungen überhaupt anstreben bzw. erreichen. Die wichtigste Bedingung, die erfüllt sein muß, ist die Verstehensbedingung; dies heißt: Strebt ein Vortragender an, daß seine Ausführungen a $k z$ e $p$ t i e $r t$ werden, muß er zunächst dafür Sorge tragen, sprachlich ver $\mathrm{s}$ ta $\mathrm{n}$ de $\mathrm{n}$ zu werden"

[vgl. im Text oben]

In den Textausschnitten (1) bis (5) geht es mir um die gesperrt gedruckten Ausdrücke akzeptieren, erfolgreich sein und versteben bzw. um ihre kotextbedingten grammatisch regelgerechten Modifikationen. Dichotomisch unterscheide ich zwischen zwei Verwendungsweisen von sprachlichen Ausdrücken: In (1) bis (3) liegen nichtwissenschaftliche Verwendungen der fraglichen Ausdrücke vor; in (4) dagegen liegen wissenschaftliche Verwendungen vor. Letzteres besagt, daß in (4) die fraglichen Ausdrücke relativ zu nominalen Festsetzungsdefinitionen verwendet sind. ${ }^{9}$ In solchen Definitionen erscheinen die nicht-wissenschaftlichen Ausdrücke im Definiendum und werden terminologisiert. ${ }^{10}$ Schließt die definitorische $\mathrm{Be}$ deutungskonstitution an den nicht-wissenschaftlichen Gebrauch der in den Definienda stehenden Ausdrücke an, dann werden durch den Akt des Definierens solche wissenschaftlichen $F$ achausdrücke geschaffen, die an die nicht-wissenschaftliche Spracherfahrung und damit an die Alltagskenntnisse der Sprachsubjekte tendenziell angeschlossen sind. Dadurch bleibt das nicht-wissenschaftliche, u.a. durch die Sprachpraxis erworbene und vermittelte Alltagswissen als Verstehensbasis auch für das Verstehen von verwendeten Fachausdrücken in Geltung. Diese Art der definitorischen Terminologisierung, die prinzipiell auf die meisten nicht-wissenschaftlichen Ausdrücke angewandt werden kann, verwirklicht konkret - ohne Rekurs auf philosophischen Schwulst und ohne den Kontext "transzendentaler Träumereien"11 - die Einsicht, daß die Bedingung der Möglichkeit für 
wissenschaftliche Erkenntnis und Erfahrung qua Wissenschaftssprache wenigstens in den Sozialwissenschaften - häufig die je erlernte nichtwissenschaftliche Sprache ist.

In (4) sind die fraglichen Ausdrücke als Fachausdrücke verwendet, in (1) bis (3) dagegen nicht. Ich fasse daher die Ausdrücke, deren Verwendung in (4) vorliegen, als Terminologisierungen derjenigen Ausdrücke auf, deren Verwendung in (1) bis (3) vorliegen. Da die Ausdrücke in (1) bis (3) anders verwendet sind als in (4), spielen sie - so möchte ich unterstellen - auch jeweils eine andere Rolle beim Zustandekommen des Textverstehens. Wenn ein Textrezipient die Ausdrücke kennt und wenn alle weiteren Verstehensvoraussetzungen gegeben sind, konstituiert sich das Textverstehen auf zwei verschiedenen Verstehensebenen: als Ergebnis einer Rezeption von (1) bis (3) wird ein nicht-wissenschaftlicher, entsprechend bei (4) ein wissenschaftlicher Sachverhalt verstanden. ${ }^{12}$ Ist nun ein beliebiger Ausdruck $A$, z.B. akzeptieren, der in einer nicht-wissenschaftlichen Sprache einen geregelten Gebrauch und damit wenigstens eine relativ bestimmte, praktisch eingespielte Bedeutung hat, in der oben erläuterten Art mittels einer Nominaldefinition terminologisiert und damit auch als wissenschaftlicher Fachausdruck definitionsgerecht verwendbar, dann ergibt sich für die Textrezipienten, seien sie nun Zuhörer oder Leser, prinzipiell die Möglichkeit, auf der einen oder der anderen Verstehensebene Verstehen zu erreichen. Auf welcher Verstehensebene die jeweiligen Verstehensbemühungen ablaufen, ist dabei vor allem abhängig vom je aktivierbaren Vorwissen der Textrezipienten. Wer mithin z.B. die neuere wissenschaftliche Diskussion um die Fachausdrücke akzeptieren, erfolgreich sein und versteben kennt ${ }^{13}$, versteht den Textausschnitt (5) auf der Folie seines individuellen Wissenraumes ${ }^{14}$ anders als einer, der die erwähnte Diskussion und die gegebenen Definitionen zufällig gerade nicht kennt. M.E. ist der von mir geäußerte Textausschnitt (5) auf beiden Verstehensebenen zu verstehen. Ich halte dies für einen Vorteil und meine, daß in bestimmten kommunikativen Situationen, beispielsweise, wenn jemand einen Vortrag vor einem - hinsichtlich der je individuellen Wissensräume als heterogen eingeschätzen - Großauditorium hält, die Einstellung, die sich manchmal in solchen relativ gedankenlosen Redefloskeln zeigt wie z. B.wie Sie ja alle wissen ... oder uns ist ja allen bekannt, daß ... dann nicht unbedingt die angemessene ist, wenn vom Vortragenden Wert darauf gelegt wird, daß die sprachlichen Ausführungen verstanden werden.

Die gerade anhand des Beispiels Nr. 1 erläuterte Strategie, sowohl die terminologische als auch die nicht-terminologische Verwendungsweise eines Ausdruckes als Sprecher zu berücksichtigen, in der Absicht, daß zwei Verstehensebenen im Adressatenkreis eröffnet werden, ist erstens 
sicherlich nur für bestimmte Textsorten und damit für bestimmte Situationen adäquat und zweitens aus verschiedenen Gründen problematisch. So wäre beispielsweise zu fragen, ob dieses Vorgehen nicht gerade Kommunikationskonflikte schafft. Dann aber wäre gleichzeitig zu fragen, welche andere Vorgehensweise unter den gegebenen Bedingungen keine Kommunikationskonflikte schaffen würde.

Nicht unmittelbar bezogen auf die in diesem Handlungsraum ${ }^{15}$ gegebenen Bedingungen möchte ich jedoch noch folgende generelleren Bemerkungen zum methodischen Rahmen für Untersuchungen des Sprachgebrauchs machen. Es scheint mir unumgänglich zu sein, daß empirisch oder quasiempirisch ${ }^{16}$ ausgelegte Untersuchungen des Sprachgebrauchs an dasjenige Erfahrungswissen anschließen, das im nicht-wissenschaftlichen Sprechen über den Sprachgebrauch bereits immer schon ausgedrückt wird bzw. werden kann. Dies bedeutet u.a.: Die sprachwissenschaftliche Analyse des Sprachgebrauchs expliziert mehr oder weniger isolierte, klar-konfuse Alltagskonzepte und überführt sie in geordnete theoretische Konzepte, die nach der Analyse als Erklärungsbasis dienen können. ${ }^{17}$ Dies heißt zugleich, daß solche theoretischen Ausdrücke, die zur Untersuchung des Sprachgebrauchs benötigt werden, insbesondere auch solche Grundprädikate wie z.B. Handlung, Bedeutung, Versteben und andere, aber auch solche Ausdrücke eines anderen Typs wie z.B. Kommunikationskonflikt, die in nicht-wissenschaftlicher Rede nicht allgemein geläufig sind, keineswegs beliebig gewählt und auch nicht $\mathrm{x}$-beliebig expliziert oder theorieintern definiert werden können. - Eine Analyse des Alltagskonzeptes 'Verstehen' z. B. kann daher an eine semantisch/pragmatische Analyse der Verwendung des sprachlichen Ausdrucks versteben und eventuell an die seiner Feldnachbarn anschließen, um danach ein theoretisches Konzept zu erarbeiten. Mit der angedeuteten Vorgehensweise kann ein wissenschaf tlicher Begriff des Verstehens an den Alltagsbegriff angeschlossen werden ${ }^{18}$; dadurch wird auch jener, von mir exemplarisch erläuterte, Einsatz zweier Verwendungsweisen des sprachlichen Ausdruckes versteben möglich. - Theoretische Begriffe, die wie angedeutet etabliert werden, verweisen stets noch auf ihren Entdeckungszusammenhang; sie sind verankert in der erworbenen Spracherfahrung und -kenntnis sowie im Sprecherbewußtsein gerade auch des Analysesubjektes; sie verweisen die Erfahrung nicht konventionalistisch in den Verifikations- bzw. Falsifikationsbereich und damit in den Begründungszusammenhang der Theorie. ${ }^{19}$ 


\section{Zum Gebrauch des Ausdruckes Kommunikationskonflikt}

Ich möchte in diesem dritten Abschnitt versuchen zu erläutern, wie ich hier den Ausdruck Kommunikationskonflikt verwenden will. Als Ergebnis dieses Versuches werden wir noch nicht einmal über einen sog. klassifikatorischen Begriff (im strengen Sinne) verfügen. ${ }^{20}$ Das Ziel meiner nachfolgenden Erläuterungen ist lediglich erstens die angestrebte Verwendung dieses Ausdruckes wenigstens partiell zu rechtfertigen und zweitens eine gewisse semantische Stabilität für die Verwendung von Kommunikationskonflikt sicherzustellen, die gerade so flexibel ( $¥$ instabil und ₹ vage!) ist, daß einerseits eine Anzahl von Beispielen problemlos als Fälle von Kommunikationskonflikten identifiziert werden können, daß aber andererseits eine Anzahl von Beispielen nicht von vornherein nicht als Kommunikationskonflikt zählt und damit aus dem Blickfeld gerät, nur weil der Gebrauch des Ausdrucks bereits festgelegt wurde.

Der erste Schritt meiner Erläuterungen soll einfach darin bestehen, daß ich eine Reihe von authentischen Beispielen aus Diskussionen unter Sprachwissenschaftlern gebe, in denen Personen in verschiedenen kommunikativen Rollen, nämlich als Diskussionsredner (DR), als Diskussionsleiter (DL), als Zwischenrufer (ZR) und als Referent ( $R$ ) mit sprachlichen Äußerungen auf Ausschnitte der Kommunikation Bezug nehmen, die eventuell als sprachlicher Ausdruck von Kommunikationskonflikten gelten können. Ich habe bewußt mehrere Beispiele hinzugenommen, die $\mathrm{m}$.E. nicht zu den klaren Fällen gehören; d.h., man wird im Falle der Analyse fragen müssen, ob es sich nicht um solche Konflikte handelt, die nur kommunikativ indiziert bzw. ausgetragen werden, nicht aber selbst durch die Kommunikation bedingt sind. 21

BEISPIEL Nr. 2 - 34

2. ZR: "Lauter bittel Hier bört man Sie nicht."

3. DL: "Wir schließen am besten die Fenster, der Bagger ist offensichtlich lauter als der Referent."

4. R: "Entschuldigen Sie bitte! Icb wurde hier am Tonband gerade abgelenkt. Können Sie Ibre Frage bitte wiederbolen?"

5. R: "Ich dacbte, diese A ngelegenbeit sei bereits geklärt und verstebe eigentlich nicht, wieso Sie jetzt erneut nach dem theoretiscben Status der semantischen Merkmale fragen."

6. R: "Icb verstebe nicht so recht, was Ibr Einwand zur Sache beitragen soll."

7. DR: "Könnten Sie mal erläutern, wie Sie den Ausdruck g e n e ri e re n gebraucht baben? Offensichtlich doch nicht im Sinne Chomskys, oder?"

8. DL: "Gestatten Sie mir die Bemerkung - aucb mit Rücksicht auf die lange Rednerliste: Icb babe den Eindruck, daß einige bier permanent aneinander vorbeireden." 
9. DR: "Ich muß gesteben, daß ich mich bier völlig mißverstanden füble."

10. DR: "Icb mache jetzt den Vorscblag, daß wir bier zum näcbsten Diskussionspunkt übergeben, denn diese frucbtlosen Haarspaltereien fübren ja doch nur zu Streitereien. Die Herren können das ja vielleicht nachber beim Bier aushandeln."

11. DR: "Icb bin ja bei den Linguisten bier nur Gast und kann daber sacblich nicbts beitragen. Als Literaturwissenscbaftler kann ich mir aber die Bemerkung nicht verkneifen..., oder vielleicht ist es besser, wenn ich es als Frage formuliere: Versteben Sie denn Ibr Fachchinesisch?"

12. R: "Ibre Ausfübrungen zeigen mir, daß Sie meine dritte These nicht ricbtig interpretiert haben. Das kann natürlich auch an der Formulierung liegen, ich gebe gerne $z u$, daß diese etwas komplex bzw. komprimiert geraten ist."

13. DL: "Das war ein bißchen viel auf einmal! Können Sie vielleicht Ibren Beitrag auf einen kurzen Nenner bringen?"

14. DR: "Was meinen Sie denn mit Praxeogram $m$ ?"

15. DR: "Wenn wir in dieser beiklen Frage weiterkommen wollen, müssen wir wobl oder übel erst einmal festlegen, was bier unter $B$ e de utung verstanden werden soll,"

16. DR: "Ibre scbönen Formeln an der Tafel, verebrter Herr Kollege, sind für mich Böbmische Dörfer. Sie müssen sich schon die Mübe macben, dies Zeugs zu übersetzen."

17. DR: "Ich babe den Eindruck, daß wir uns bier deswegen so scbwer tun, weil jeder unter Transformation etwas anderes verstebt."

18. R: "Die Richtung Ibrer Frage ist mir eigentlich nicht ganz klar."

19. R: "Icb danke Ibnen für Ibren ausfübrlichen Diskussionsheitrag, habe aber nicht den Eindruck, daß Sie micb nacb etwas gefragt baben."

20. DR: "Zunächst zwei Verständnisfragen."

21. DR: "Sollte das eine empirische Feststellung, eine Hypothese oder gar eine Definition sein?"

22. R: "Gegen welcben Punkt meiner A usfübrungen baben Sie eigentlich argumentiert?"

23. R: 'Ibre Fragen zeigen mir, daß ich mich präzisieren muß.'

24. DR: "Mir ist eigentlich nicht ganz klar geworden, in welchem Sinne Sie von Eige n n a me $n$ reden."

25. DR: "Ich bin mir nicht ganz sicher, ab ich Sie in einem zentralen Punkt richtig verstanden babe. Verwenden Sie die Termini $S$ ig $n$ ifik at ion $s$ relation und $R$ eferenzre lat ion synonym oder nicht? Wenn nicht: wo liegt der Unterschied?"

26. R: "Lieber Herr $X$, wir kennen uns schon so lange und baben ja auch schon öfters gerade über diesen Punkt diskutiert, daß ich Ihnen zu Ibrer Frage zum Status von Traumwelten nur sagen kann: Entweder haben Sie vorbin geträumt, oder Sie wollen mich ärgern." 
27. R: "Auf Ibre Frage kann icb nur mit einer Gegenfrage antworten: Sind wir bier eigentlich im Prose minar oder in einer wissenscbaftlichen Diskussion?"

28. $\mathrm{DR}_{1}$ : "Schließlich müssen wir doch davon ausgeben, daß jedes spracbliche Zeicben aus einem signifikant und einem signifié bestebt."

$\mathrm{DR}_{2}$ : "Das brauchen Sie doch mir nicht zu sagen!"

29. DR: "Ibre A usfübrungen baben mich vollkommen überzeugt. Icb darf Sie auf meinen A ufsatz " $X$ " binweisen, in dem icb bercits vor 9 Jabren genau die gleiche Etymologie entwickelt babe."

30. DR: "Dem jungen Kollegen auf einer der binteren Bänke möcbte icb zu seinem letzten Beitrag noch sagen: Mit Ironie sind noch keine wissenschafticben Probleme gelöst worden."

ZR: "Mit tieriscbem Ernst und Dogmatismus aber aucb nicht!"

DL: "Sie baben nicbt das Wort. Außerdem wollen wir bier sacblicb diskutieren!"

31. DL: "Herr Kollege X, Sie waren angesprocben. Wollen Sie dazu Stellung nebmen?"

$\mathrm{DR}_{\mathrm{X}}$ : "Ich glaube nicht, daß sicb das lobnt,"

32. R: "Icb bedanke mich für die Belebrung. Es ist schön, daß wir so gebildete Leute unter uns baben, die es immer wieder fertig bringen, uns von den eigentlicben Problemen so charmant abzulenken."

33. $\mathrm{R}$ : "Um allen $M_{\imath} \beta$ verständnissen von vornbere in vorzubeugen, möcbte ich einleitend folgendes klarstellen: Icb werde strikt zwiscben Normen und Regeln unterscheiden. Für mich sind Normen lediglich diejenigen Regeln, die vorgeschrieben werden."

34. DR: “Wenn Sie glauben, icb bätte die Ricbtung lbrer Kritik nicbt verstanden, dann irren Sie sich. Ich verstebe nur Sie nicht, wenn ich daran denke, was gerade Sie vor ein paarMonaten dazu gesagt baben."

Für einen teilnehmenden Beobachter, der wissenschaftliche Diskussionen unter dem Aspekt ihrer Konfliktträchtigkeit untersuchen will, sind solche Textstellen, wie die Beispiele Nr. 2 bis Nr. 34, mögliche Ansatzpunkte für eine Untersuchung. Ich will sie Konfliktindikatoren nennen. Eine erste Aufgabe wird sein, diese in irgendeiner Weise zu ordnen. Eine solche Ordnung ist dann brauchbar, wenn sie ihren Zweck erfüllt; um sie herzustellen, benötigt man wenigstens ein zweckgerechtes Ordnungskriterium.

In einem zweiten Schritt meiner Erläuterungen zur Verwendung des Ausdruckes Kommunikationskonflikt will ich versuchen, ein vorläufiges Kriterium dadurch zu bestimmen, daß ich ein vorläufiges Interpretament für Kommunikationskonflikt angebe. ${ }^{22}$ Einerseits kann man einen theoretisch isolierten Ausdruck wie Kommunikationskonflikt nicht einfach definieren, andererseits scheint mir eine theoriebezogene Definition, die prinzipiell entgegen der nichtwissenschaftlichen Verwendung des zu definierenden Ausdruckes vorgenommen wird, ein inhaltlich nicht sehr interes- 
santes Ergebnis eines wissenschaftsdogmatischen Willküraktes zu sein. Der Gebrauch der Ausdrücke Konflikt und Kommunikation ist bildungssprachlich bereits mehr oder weniger eingespielt. Mithin kann über die Semantik der beiden Ausdrücke keineswegs beliebig verfügt werden, und daher ist auch das Kompositum Kommunikationskonflikt bereits semantisch motiviert. - Wortbildungsmäßig entspricht er Ausdrücken wie $G e$ nerationenkonflikt, Rollenkonflikt, Proporzkonflikt, Klassenkonflikt, Interessenkonflikt u.a. Untersucht man die Verwendung dieser Komposita nicht etwa in fachwissenschaftlichen, sondern in nicht-wissenschaftlichen, insbesondere in bildungssprachlichen Texten und nimmt den Ausdruck Konflikt sowie solche Fügungen wie bewaffneter Konflikt, tragiscber Konflikt, sozialer Konflikt u.a. hinzu, dann erhält man etwa folgendes vorläufige Interpretament ${ }^{23}$ : Der Ausdruck Konflikt, die Komposita vom gezeigten Typ sowie die erwähnten Fügungen werden verwendet, um auf Zustände oder Prozesse Bezug zu nehmen, die dann auftreten, wenn wenigstens zwei miteinander nicht verträgliche Verhaltens- oder Handlungstendenzen bzw. -ziele gemeinsam in einem Interaktionszusammenhang vorkommen und wenn auf die dadurch gegebene Konstellation in irgendeiner Weise reagiert wird. - Alle Komposita vom Typ X-KONFLIKT stehen zum Ausdruck Konflikt in der lexikalisch-semantischen Relation der Unterordnung, d.h.: sie sind Hyponyme zu Konflikt. Will man mithin nicht von vornherein mehr oder weniger willkürliche definitorische Festsetzungen treffen, die den Ausdruck Kommunikationskonflikt möglicherweise semantisch weitgehend von seinen Feldnachbarn isolieren, dann sollte man das gerade erwähnte feldkonstitutive Interpretament für Konflikt und Komposita vom Typ X-KONFLIKT bei einer eventuellen Terminologisierung von Kommunikationskonflikt möglichst berücksichtigen. Man hat so auch die Möglichkeit - im Sinne der Erläuterungen im Abschnitt 2-Kommunikationskonflikt so zu verwenden, daß er auf zwei Verstehensebenen verstanden werden kann.

Ich werde daher - unter Berücksichtigung des Interpretamentes für den Ausdruck Konflikt - den dazu hyponymen Ausdruck Kommunikationskonflikt verwenden, um auf Prozesse Bezug zu nehmen, die dann auftreten, wenn zwei nicht verträgliche kommunikative Ziele bzw. kommunikative Absichten in einem Interaktionszusammenhang vorkommen (und miteinander erkennbar kollidieren) und wenn auf die dadurch gegebene Konstellation in irgendeiner Weise kommunikativ reagiert wird. In einem dritten Schritt meiner Erläuterungen zur Verwendung von Kommunikationskonflikt möchte ich das soeben gegebene Interpretament für Kommunikationskonflikt anhand einer partiellen Analyse eines Beispiels konkretisieren und etwas präzisieren. 
BEISPIEL Nr. 35

Objektiv-äußere Gegebenheiten des Handlungsraums: großer Vortragssaal; $\mathrm{DR}_{1}$ sitzt ganz hinten, $\mathrm{DR}_{2}$ ganz vorne.

$\mathrm{DR}_{1}$ und $\mathrm{DR}_{2}$ konstituieren, indem sie beginnen, miteinander zu diskutieren, einen Interaktionsraum. 24

Textausschnitt: Beginn der Diskussion

$\mathrm{DR}_{1}$ : (1) "Ich möcbte Herm $X_{\mathrm{DR}_{2} \text { fragen, ob er den Begriff des Diaphonems }}$
im Sinne des Phonologie-Grafen bzw. Papstes verstebt."

$\mathrm{DR}_{2}$ : (2) "Icb habe Sie eben akustisch nicbt verstanden."

$\mathrm{DR}_{1}$ : (3) "Icb habe Sie eben gefragt, ob Sie den Begriff des Diaphonems im Sinne von Trubetakoy versteben."

Es handelt sich um ein sehr einfaches Beispiel, das lediglich in einer einfachen Verschriftlichung vorliegt, was für den hier gegebenen Zweck m.E. kein Handicap darstellt. Selbst wenn man nur eine eingeschränkte Analyse dieses Textausschnittes anstrebt, muß man erstens eine ganze Reihe von methodischen Voraussetzungen machen, die zusammen genommen eine methodische Position markieren (sollten). Zu dieser möchte ich nur eine kurze Bemerkung machen. Diese Position kann m.E. nur eine hermeneutische sein; das soll hier wenigstens heißen: Bevor ein Analysator beginnen kann, den vor seinen Erkenntnisapparat gebrachten Text im Lichte theoretischer Begrifflichkeit und relativ zu gesetzten Untersuchungszwecken zu analysieren, muß der Text auf einer nicht-wissenschaftlichen Verstehensebene mehr oder weniger, im günstigsten Fall möglichst weitgehend, verstanden sein. Dieses letztere Verstehen ist ein solches vom extrakommunikativen Standpunkt aus; verglichen mit dem angestrebten wissenschaftlichen, d.h. theoriebezogenen Verstehen ist es als Vor-Verstehen charakterisierbar und darf nicht mit dem Verstehen verwechselt werden, das die Kommunizierenden selbst (hier $\mathrm{DR}_{1}, \mathrm{DR}_{2}$ ) intrakommunikativ erreichen. Es muß einerseits stets eine Differenz, das heißt ein Mehr oder Weniger, in Kauf genommen werden; andererseits aber kann auch hier mit der Regelhaftigkeit und der Verbindlichkeit der je verwendeten Sprache gerechnet und damit auch eine gewisse Gleichartigkeit der Welterfahrung unterstellt werden. Das an die vorgängige sprachliche Praxis und Welterfahrung anknüpfende Vor-Verstehen kann in der Analysetätigkeit des Analysators nicht säuberlich vom wissenschaftlichen Verstehen getrennt werden, insbesondere dann nicht, wenn es um Fragen geht, die auch den Sinn des Textes betreffen. Das Vor-Verstehen geht, unter Umständen kontrollierbar, in das wissenschaftliche Verstehen ein; ein Sachverhalt, angesichts dessen Sozialwissenschaftler manchmal zu dem Glauben neigen, sie befänden sich, weil sie vergleichend auf die sog. Naturwissenschaftler schielen, in einem permanenten Rechtfertigungszwang. Ich bin 
dagegen umgekehrt der Meinung, daß, falls Naturwissenschaftler behaupten, sie hätten es meistens mit nicht-vorverstandenen Gegenständen zu tun, ihrerseits zu rechtfertigen haben, wie sie zu dieser Behauptung kommen. - Zweitens benötigt man - auch zur Analyse eines so einfachen Beispiels wie Nr. 35-einen theoretischen Rahmen, in dem die verwendeten Fachausd rücke wenigstens als vorläufig definierte gelten. Ein solcher Bezugsrahmen ist gegeben. Er kann (und braucht) hier nicht erst entwickelt zu werden. ${ }^{25}$ Seine Konturen können aus der Analyse selbst erschlossen werden; wo das nicht möglich ist, setze ich auf Evidenz.

Ich versuche zunächst vor allem anhand des Beispiels Nr. 35 und zum Teil unter Bezugnahme auf die Beispielgruppe Nr. 2 bis Nr. 34 folgende erste Frage zu beantworten: Was soll - in dem vorgeschlagenen Interpretament für den Ausdruck Kommunikationskonflikt - heißen, daß zwei nicht verträgliche kommunikative Ziele bzw. kommunikative Absichten in einem Interaktionszusammenhang erkennbar miteinander kollidieren?

Das Beispiel Nr. 35 ist ein sprachlicher Ausschnitt aus einer öffentlichen, wissenschaftlichen Diskussion. Eine solche Diskussion ist eine spezifische Form des Gesprächs. ${ }^{26}$ (1), (2) und (3) sind Gesprächsschritte. Jeder Gesprächsschritt besteht hier aus gerade einer Satzäußerung. Per Interpretation kann hier jeder Satzäußerung gerade eine Sprechhandlung zugeordnet werden. - A posteriori nehme ich an, daß in einem Zwei-Personen-Gespräch wenigstens ein Gesprächsteilnehmer ein kommunikatives Gesprächsziel erreichen will. Solche Gesprächsziele müssen vor oder zu Gesprächsbeginn keineswegs immer klar konturiert sein; sie können sich im Gesprächsverlauf erst herausbilden bzw. modifizieren.

Wer ein Gesprächsziel erreichen will, muß Sprechhandlungen vollziehen. Eine Sprechhandlung ist kategorial durch ihr kommunikatives Ziel bestimmbar. Das kommunikative Ziel einer Sprechhandlung kann Unterziel auf dem Wege zur Erreichung des Gesprächsziels sein. Welches kommunikative Ziel ein Gesprächsteilnehmer mit dem Vollzug einer Sprechhandlung auch immer erreichen will, er muß simultan stets die kommunikative Absicht haben, daß er - je nach gegebenem Fall - vom jeweils Angesprochenen und/oder von den Zuhörern sprachlich verstanden wird. Die kommunikative Absicht, Sprachverstehen ${ }^{27}$ zu erreichen, ist kommunikationslogisch - und kommunikationslogisch ist für mich nicht das gleiche wie manipulationslogisch - eine notwendige Bedingung, um kommunikative Ziele erreichen zu können. - Ich gehe weiterhin a posteriori davon aus, daß - wenigstens im Falle der face-to-face-communication - der je angesprochene Gesprächsteilnehmer gesprächsbereit ist und daher seinerseits die kommunikative Absicht hat, das sprachlich zu verstehen, was der Ge- 
sprächspartner geäußert hat. Dies bedeutet: ich berücksichtige hier Fälle von Kommunikationsverweigerungen nicht. E in Fall von expliziter Kommunikationsverweigerung liegt im Beispiel Nr. 31 vor. -

Mit dem gesprächseröffnenden Gesprächsschritt (1) hat DR 1 - sog. Ernsthaftigkeit und Aufrichtigkeit vorausgesetzt - wenigstens das kommunikative Ziel verfolgt, vom angesprochenen $\mathrm{DR}_{2}$ auf seine gestellte Frage eine Antwort zu bekommen. Wenn $\mathrm{DR}_{1}$ dieses kommunikative Ziel hatte, muß - gemäß erläuterter Annahme - weiterhin unterstellt werden, daß $\mathrm{DR}_{1}$ simultan die kommunikative Absicht hatte, daß seine Äußerung (1) von $\mathrm{DR}_{2}$ als Frage nach etwas verstanden wird. Wie jedoch der Gesprächsschritt (2) zeigt, konnte $\mathrm{DR}_{1}$ diese seine Absicht nicht verwirklichen, mithin sein kommunikatives $Z$ iel nicht erreichen. Oder anders ausgedrückt: Der mit dem Gesprächsschritt (2) beginnende, für den Analysator wahrnehmbare Teil der kommunikativen Nachgeschichte der in (1) ausgedrückten Sprechhandlung zeigt, daß diese verbale Fragehandlung im konstituierten Interaktionsraum und nur in diesem und nicht etwa im gesamten Handlungsraum nicht erfolgreich war. Man sollte aber bei dieser überwiegend sprecherorientierten Interpretation nicht stehen bleiben! Denn unter der ebenfalls bereits genannten Annahme, daß der Angesprochene, hier mithin $\mathrm{DR}_{2}$, (auch deswegen, weil er damit rechnen muß, selbst verpflichtet zu sein, die kommunikative Rolle des Sprechers zu übernehmen), die kommunikative Absicht hat, die vom Gesprächspartner gemachte Äußerung zu verstehen, zeigt (2), daß DR 2 das kommunikative $Z$ iel von $D_{1}$ nicht erschließen konnte, da er (1) akustisch nicht voll verstanden hat und mithin seine kommunikative Absicht, (1) sprachlich zu verstehen, nicht verwirklichen konnte. Dies bedeutet demnach: Auch der kognitive Prozeß, den $\mathrm{DR}_{2}$ in der kommunikativen Rolle des angesprochenen Hörers in Gang gesetzt hat, war auf der ersten Ebene nicht erfolgreich. ${ }^{28}$

Bezogen auf das oben angegebene Interpretament für den Ausdruck Kommunikationskonflikt kann man nun zunächst folgendes feststellen: Es verträgt sich nicht miteinander, daß einer der Gesprächsteilnehmer in der kommunikativen Rolle des Sprechers, hier DR 1 , ein kommunikatives Ziel hat, hier eine Antwort auf eine Frage zu bekommen, und daß ein anderer Gesprächsteilnehmer in der kommunikativen Rolle des angesprochenen Hörers, hier DR 2, die kommunikative Absicht hat, zum Erreichen eines Sprecherzieles einen kognitiven Prozeß in Gang zu setzen, der zum Verstehen einer Äußerung des anderen, hier des Gesprächsschrittes (1), führen soll, in der Verwirklichung seiner Verstehensabsicht jedoch gehindert wird, derart, daß er Sprachverstehen nicht erreichen kann. Verkürzt und generalisiert heißt das, als Antwort auf die erste gestellte Frage: Das kommunikative Ziel des Sprechers kollidiert erkenn- 
bar mit der kommunikativen Absicht des Hörers. Beide sind in ihrem kommunikativen Wollen gleichermaßen geschädigt, nicht etwa nur der Sprecher. Liegt eine solche Konstellation in einem Interaktionsraum vor, oder anders ausgedrückt: ist ein konstitutierter Interaktionsprozeß innerhalb eines Zeitintervalls derart strukturiert, spreche ich von einem a kut en Kommunikat ion skonflik t. Akut sollen diejenigen Kommunikationskonflikte heißen, die von den Beteiligten unmittelbar, nachdem sie bemerkt wurden, verbal thematisiert werden, so daß für die Gesprächsteilnehmer und den Analysator ein sprachlicher Konfliktindikator gegeben ist. Einen Untertyp der akuten Kommunikationskonflikte bilden die S p r a ch verst e he n skonflikte; im Beispiel Nr. 35, aber auch in den Beispielen Nr. 14, 17, 25 u.a. liegen Fälle vor, die zu diesem Typ gehören.

Gerade wenn Sprachverstehenskonflikte vorliegen bzw. insbesondere dann, wenn man den Typ der Sprachverstehenskonflikte, der seinerseits in eine Reihe von Untertypen differenziert werden kann, untersucht, wird deutlich, daß beide Gesprächsteilnehmer wechselseitig aufeinander angewiesen sind. Eine vollzogene Sprechhandlung kann daher nur dann auf der ersten Ebene unmittelbar erfolgreich sein, wenn der noch während ihres Vollzugs einsetzende kognitive Prozeß des Angesprochenen wenigstens zum Verstehen der Sprechhandlung führt. Die koordinierten Aktivitäten der beiden Partner lassen sich daher auch als konstitutive Teile einer übergeordneten Gemeinschaftshandlung auffassen, deren Ziel die koagierenden Individuen nur gemeinsam erreichen können. ${ }^{29}$

Bezogen auf das vorgeschlagene Interpretament für den generischen Ausdruck Kommunikationskonflikt sei nun noch eine zweite Frage gestellt: Was soll heißen, daß in irgendeiner Weise kommunikativ reagiert wird? $\mathrm{DR}_{2}$ reagiert, indem er (2) Ich babe Sie eben akustiscb nicht verstanden äußert, kommunikativ auf den ersten Gesprächsschritt. Falls (2) vom Angesprochenen, und übrigens auch von einem teilnehmenden Beobachter, richtig verstanden worden ist, gilt (2) als Konfliktindikator, und zwar hier als einer, der die Konfliktursache selbst benennt, was keineswegs von allen Typen von Konfliktindikatoren gesagt werden kann. (2) ist eine Äußerung, die eine Aufforderung ausdrückt, nämlich die, (1) wenigstens sinngemäß zu wiederholen. (3) zeigt, daß (2) von $\mathrm{DR}_{1}$ als gerade diese Aufforderung verstanden worden ist. - (2) ist eine konfliktindizierende Äußerung, die eine kontrakonfliktäre ${ }^{30}$ Sprechhandlung ausdrückt, und zwar eine Aufforderungshandlung mit dem metakommunikativen Ziel, die Beseitigung des aufgetretenen Sprachverstehenskonfliktes einzuleiten. Dies geschieht dadurch, daß DR 2 mit (2) in den gegebenen Interaktionsraum wenigstens eine neue Interaktionsbedingung einführt. ${ }^{31}$ Sie besteht darin, daß dem 
Angesprochenen $D_{1}$ die Verpflichtung auferlegt wird, seine Äußerung (1) wenigstens sinngemäß zu wiederholen. Die Redeweise von "kommunikativ reagieren" im Interpretament zu Kommunikationskonflikt ist mithin nicht so zu verstehen, daß die konfliktindizierenden Äußerungen reaktive Sprechhandlungen ausdrücken. Dies kann auch der Fall sein.

Häufiger ist jedoch - nach meinen Beobachtungen - daß kontrakonfliktäre Sprechhandlungen initiativ sind, d.h. durch Einführung wenigstens einer neuen Interaktionsbedingung eine eingebettete Handlungssequenz eröffnen. ${ }^{32}$ Dies ist auch im Beispiel Nr. 35 der Fall. Mit der in (2) ausgedrückten kontrakonfliktären Aufforderung eröff net $\mathrm{DR}_{2}$ eine eingebettete metakommunikative Handlungssequenz. Mit (2) spricht $\mathrm{DR}_{2}$ ü b e r die von ihm nicht vollständig verstandene Äußerung (1). Das Faktum, daß mit (2) gesprächsintern ü b e r eine Äußerung desjenigen Gesprächs gesprochen wird, zu der (2) selbst gehört, ist aber nur eine notwendige Bedingung dafür, daß man mit guten Gründen von Metakommunikation eines bestimmten Typs sprechen kann. Die nachfolgenden hinreichenden Bedingungen charakterisieren gerade diesen Typ. Das Sprechenüber muß das auf diejenige Kommunikation, in die es eingebettet ist, bezogene Ziel haben, Sprachverstehenskonflikte entweder prophylaktisch zu verhindern oder im Nachhinein - wie im Beispiel Nr. 35 - zu beseitigen. Bei diesem Typ handelt es sich um kooperativ angelegte Metakommunikation. ${ }^{33}$ Diese ist um den kommunikativen Erfolg der übergeordneten Gemeinschaftshandlung bemüht.

Damit ist angedeutet, daß die Redeweise "in irgendeiner Weise kommunikativ reagieren" ganz bewußt möglichst allgemein verstanden werden und z.B. auch initiative und metakommunikative Sprechakte einbegreifen soll. Damit möchte ich die exemplarischen Erläurerungen zur Verwendung des Ausdruckes Kommunikationskonflikt abbrechen.

Die bisher versuchte Analyse des Beispiels Nr. 35 ist noch sehr grob; daher noch einige Bemerkungen dazu. Wenn auch die mit (1) vollzogene Sprechhandlung als solche nicht unmittelbar erfolgreich war, so kann dennoch nicht gesagt werden, daß $D R_{1}$ mit (1) die Gesprächseröffnung nicht gelungen ist, denn mit (2) gibt $\mathrm{DR}_{2}$ kooperativ zu verstehen, daß er gesprächsbereit ist. Wie (3), einf ach deswegen, weil (3) vollzogen wurde, zeigt, geht auch $\mathrm{DR}_{1}$ davon aus, daß er sich mit $\mathrm{DR}_{2}$ bereits im Gesprächszustand befindet. Hier zeigt sich nun, daß die korrekte Anwendung des Prädikats nicht erfolgreich sein (auf der ersten, der Verstebensebene) a uf Sprechakte keineswegs schon besagt, daß ein Gesprächsschritt kommunikativ vollständig erfolglos vollzogen wurde. (2) zeigt außerdem, daß $\mathrm{DR}_{2}$ (1) zumindest soweit verstanden haben muß, daß er erschließen konnte, daß er von $D_{1}$ angesprochen wurde, d.h. $\mathrm{DR}_{2}$ muß wenigstens 
seinen - im Text als $X_{D_{2}}$ wiedergegebenen - Namen verstanden haben. Weiterhin ist bemerkenswert, wie DR 1 (3) verglichen mit (1) formuliert. Er ersetzt nämlich des Pbonologie-Grafen bzw. Papstes in (1) in (3) durch von Trubetzkoy. An diese Ersetzung in (3) kann freilich keine intersubjektiv verifizierbare interpreta torische Aussage, wohl aber eine - wie mir scheint fruchtbare - interpretatorische Vermutung folgenden Inhalts angeschlossen werden: $D_{1}$ unterstellt, daß $\mathrm{DR}_{2}$ eventuell deswegen Sprachverstehen nicht voll erreichen konnte, weil er nicht erschließen konnte, wer mit Phonologie-Graf in (1) gemeint war, daß mithin (2) nicht wörtlich zu nehmen ist, sondern von $\mathrm{DR}_{2}$ verwendet wurde, um erstens Zeit zu gewinnen und um zweitens eben dies als eine der Voraussetzungen zur Beantwortung der Frage herauszubekommen. Nach meiner Erfahrung wird in Diskussionen unter Wissenschaftlern der geläufige Satz Icb babe Sie (eben) akustiscb nicbt verstanden gespräch sstrategisch durchaus so verwendet. - Trotz dieser Überlegungen möchte ich aber dabei bleiben, den im Beispiel Nr. 35 indizierten akuten Kommunikationskonflikt als Sprachverstehenskonflikt, bedingt durch partielle Kanalstörung, aufzufassen.

\section{Kommunikationskonflikte und die Verwendung von Fachausdrücken}

Im Folgenden muß ich eine ganze Reihe verschiedener Typen von Kommunikationskonflikten ausschließen, und zwar z.B. diese:

(1) alle Typen von kanalbedingten Störungen,

(2) alle Typen, die syntaktisch bedingt sind, und zwar sowohl solche, die durch abweichende als auch solche, die durch zu komplizierte Textkonstruktion zustande kommen,

(3) alle Typen, die akut gestörte oder durch nachlassende Aufmerksamkeit im Interaktions- oder Handlungsraum zustande kommen können,

(4) alle Typen, die durch unterschiedliche Interpunktion des Gesprächsverlaufes entstehen können,

(5) alle Typen, die durch implizite oder explizite Partnerbewertungen bzw. Selbst- und Partnereinschätzungen sich entwickeln können,

(6) alle gesprächsstrategisch bedingten Typen, eingeschlossen solche, die durch Nichtbeachtung von Konversationsmaximen zustande kommen. ${ }^{34}$

Weiterhin gehe ich nicht näher auf die zahlreichen Typen von Kommunikationskonflikten ein, die von den beteiligten Kommunikationspartnern 
nicht sprachlich indiziert werden. Fälle, die zu diesem Typ gehören, lassen sich als teilnehmender Beobachter nicht analysieren. Um solche gesprächsintern nicht verbalisierten Konflikte zu studieren, muß man um hinreichend gesicherte Ergebnisse erzielen zu können - die Komm nikationsteilnehmer im Nachhinein z.B. gezielt befragen. Solche Untersuchungen sind schon gemacht worden, und zwar besonders hinsichtlich der Sprachkommunikation zwischen Laien und Fachwissenschaftlern. Ich nenne nur eine Untersuchung, und zwar die von Dubach und von von Rechenberg, durchgeführt in der Medizinischen Universitäts-Polyklinik in Basel. ${ }^{35} 88$ Patienten wurde die Diagnose ausführlich erklärt. Nach kurzer Zeit dazu befragt, hatten nur noch 76 ein sog. Krankheitsverständnis, und zwar entlang einer Bewertungsskala : $55 \%$ ein gutes, $26 \%$ ein mittelmäßiges und $19 \%$ ein schlechtes. 5 Patienten hatten den Namen ihrer Krankheit vergessen, 3 gaben eine total andere Diagnose an, 5 erklärten - trotz gegenteiliger Arztaussagen - sie seien völlig gesund. Es gab zahlreiche, geradezu unglaubliche Kommunikationskonflikte; nur ein schönes Beispiel: Bei einer Patientin diagnostizierte der Arzt funktionelle Bauchbeschwerden. Nach ihrer Krankheit befragt, gab diese Patientin an, der Arzt habe ihr erklärt, sie leide unter Heuschnupfen! Ein Test ergab, daß einer der diagnostizierenden Arzte in $68 \mathrm{~F}$ ällen angab, er sei vollkommen davon überzeugt, daß der Patient seine Erklärungen vollständig verstanden hätte. Der Gegentest ergab jedoch, daß dies nur in 29 Fällen zutraf. Ein weiterer Test, der die Patienten nach den Gründen der Kommunikationskonflikte befragte, ergab hochsignifikant: Es sind die medizinischen Fachausdrücke, die die Hauptursache für die Sprachverstehenskonflikte sind. Dies entspricht auch den Ergebnissen von Untersuchungen aus anderen Kommunikationsbereichen, in denen Laien mit Fachleuten kommunizieren. Meine Beobachtungen zur Verwendung von wissenschaftssprachlichen Ausdrücken in den Sozialwissenschaften haben bisher ergeben, daß auch in fachinternen und in der interfachlichen Sprachkommunikation die $\mathrm{V}$ e r w e n d u n $\mathrm{g}$ von Fachausdrücken (nicht die Fachausdrücke!) der häufigste kommunikative Störfaktor ist. ${ }^{36}$ Aus diesem Grunde werde ich nachfolgend auf Sprachverstehenskonflikte eingehen, die durch verwendete Fachausdrücke entstehen können. Es handelt sich allerdings nur um Übersichtsbemerkungen. -

Bisher habe ich den Ausdruck Facbausdruck generisch verwendet, um unspezifisch auf alle Arten von sprachlichen Fachausdrücken Bezug nehmen zu können, die in irgendeinem Fach fachspezifisch verwendet werden. Alle Ausdrücke der folgenden Liste sind Fachausdrücke. 


\section{LISTE VON FACHAUSDRUCKEN}

Aus der Linguistik:

Phonologisch determiniertes Allomorph, distinktives Merkmal, Hyponymierelation, Basisregel, kontextsensitive Subkategorisierungsregel, strikte Subkategorisierungsregel, Regelformulierung, grammatische Regularitäten, privative Opposition, Systemlinguist, taxonomischer Strukturalismus, nordamerikaniscber Deskriptivismus, Apokoinu, Apokope, Transformation, Konjunktion, Palindrom, Parisyllabum, Bedeutung, Wort, performatives Verb, Sprache, Langue, ¿parole, assertiertes Präsuppositionsgefüge, analytischer Satz, Chomsky-Adjunktion, Rückwärtspronominalisierung, IC-Analyse, Jota-Operator, G-Quantor, P-Marker, Strichpunkt, Lautverscbiebung, Isolex, Wurzel, Stamm, Graph, Graphem, Distingem, Kompetenz, System, Struktur, dominieren, Knoten, Professionalismus, Halbterminus, Jargonisierung, formalisieren, generieren, empirisch, Pragmatisierung, Personenfokusierung, Gesprächsakt.

Aus anderen Fachgebieten:

DLG-Futter, T-Träger, X-Nabt, Nebelwerfer, Einsteinium, Newtonscbe Ringe, Engländer, Nortonscbwinge, Pak, Scbwarzwälder Fücbse, Fixkosten, naßscbleifen, Lizenzgeber, punktschweißen, Glüblampe, Leuchte, Flop, esox lucius, bakterielle Dysenterie, autophysiscbe Orientierung, Hexe, Rechner, Teilmantelgescboß, Lunte, krankscbießen, REFA-Lebrgang, desiderium naturale, Polylemmá, Immanenzpositivismus, Brucin, Mikrofarad, M-5-Metbode.

Ich habe mich bemüht, diese Liste so zusammenzustellen, daß möglichst viele Arten von Fachausdrücken darin vorkommen. Uber einer solchen Liste kann man verschiedene Typologien aufstellen. Diese fallen - je nach gerade gewähltem Typologiekriterium - anders aus. Mögliche Kriterien sind z. B. die folgenden:

(1) Konstitution der Bedeutung des Fachausdruckes,

(2) Gebildetheit der Formseite des Fachausdruckes,

(3) Zugehörigkeit zu einer sog. Fachsprachenschicht im Sinne der sog. vertikalen Gliederung,

(4) Semantische Motivation,

(5) Fachausdrucksbildung als Möglichkeit der Erweiterung von Fachausdrucksinventaren.

Diese Kriterien (1) bis (5) haben alle etwas mit der Bedeutung von Fachausdrücken zu tun. ${ }^{37}$ Stellt man diese Typologien auf, dann kann man feststellen, daß jede semantische Eigenschaft bzw. Eigenschaftsgruppe, die einen bestimmten Fachausdruckstyp konstituiert, zu einem anderen Typ von Sprachverstehenskonflikten führen kann. Solche Kommunikationskonflikte werde ich - der Kürze halber - fachsemantisch bedingte Sprachverstehenskonflikte nennen. 
Im Folgenden kann ich nur einige Fälle behandeln anhand einer Typologie nach dem Kriterium (1).

Dazu gebe ich zunächst eine intensionale Festsetzungsdefinition des Ausdruckes Fachausdruck. Ein Fachausdruck ist jeder sprachliche Ausdruck, der innerhalb eines Faches für die Sprecher der Fachsprache, zu der dieser Fachausdruck gehört, wenigstens eine Bedeutung hat, die er für Personen, die diese Fachsprache oder einen bestimmten Ausschnitt aus ihr nicht beherrschen, nicht hat. - Die Liste von Fachausdrücken ist ein Ausschnitt der Extension dieser Definition. Danach kann ein Fachausdruck innerhalb eines Faches erstens mehrere Bedeutungen haben, z.B. die sprachwissenschaftlichen Fachausdrücke Transformation, Konjunktion, Kompetenz. Zweitens kann ein Fachausdruck in verschiedenen Fächern verschiedene Bedeutungen haben, z.B. dominieren in der Linguistik und Biologie. Die Formulierung Bedeutung baben in der Definition soll hier aufgefaßt werden als einen bestimmten semantischen Gebrauch in einer Fachsprache haben.

Eine Typologie von Fachausdrücken nach (1) hat folgende Form:

\section{TYPOLOGIE VON FACHAUSDRUCKEN}

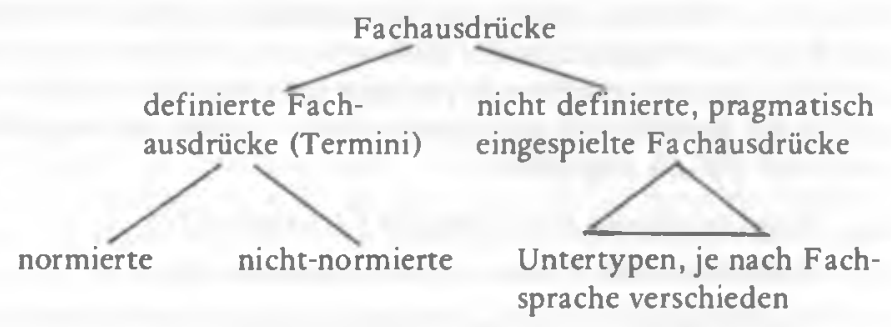

Zunächst einige Bemerkungen zu den fachsemantisch bedingten Sprachverstehenskonflikten, die durch die Verwendung nicht-normierter, definierter Fachausdrücke entstehen können. Einen Fachausdruck dieses Typs fachsemantisch korrekt zu verwenden, heißt, ihn gemäß einer semantischen Festsetzungsdefinition referierend oder prädizierend zu verwenden. ${ }^{38}$ - Denken wir uns irgendein fachinternes Gespräch zwischen zwei Wissenschaftlern über eine Problemlage ihres Faches mit dem Ziel, das Problem möglichst zu klären! Wer will, kann sich in seiner Phantasie durch die nachfolgenden Gesprächsausschnitte leiten lassen, denen einige Erläuterungen beigegeben sind. 
BEISPIEL Nr. 36

$F=$ Fachausdruck, um den es geht

1. Gesprächsausschnitt:

A: (1) "Wir baben jetzt, im Anschluß an Mates, sieben möglicbe Übersetzungen für die Feststellung $D$ ie $A$ us sage $A$ is $t$ a n aly $t$ is $c b$. Jetzt käme es darauf an zu prifen, welche der Obersetzungen dem Begriff des $g$ ra $m$ mat is cben Satzes (F) im Sinne Wittgensteins am näcbsten kommt."

B : (2) "Icb kenne zwar den Begriff des grammatiscben Satzes aus der TG, nicbt aber im Sinne Wittgensteins."

[Es folgt eine längere Dialogpartie]

2. Gesprächsausschnitt:

A: (1) "Die Frage der lediglicb beuristischen

Geschlossenbeit eines Parudigmas

stellt sicb natürlich im Falle
der Gramme me $(F)$ nicht, $\quad \rightarrow \quad\left\{\begin{array}{l}\text { Verwendung eines } \\ \text { definierten Fach- }\end{array}\right.$

denn...

Lausdruckes

B : (2) "Kurze Zwiscbenfrage: Sind Gram- (kontrakonfliktäre meme grammatische Morpheme?" $\} \backslash\{$ Frage mit hypothetischer Vermutung zu möglicher Semantisierung

A (3) "Ja ungefäbr! Es sind die kleinsten , fonfliktlösende FestSigneme, d.b. diejenigen Moneme, die - bezogen auf ein Spracbstudium - in einem gescblossenen Paradigma steben."

3. Gesprächsausschnitt:

A : (1) "Seitdem Hans im Stab ist, kommt er fast jeden Abend später aus dem Dienst; er muß meistens nocb zu einem Briefing $(F)$, wie er das\} $\rightarrow\left\{\begin{array}{l}\text { Verwendung eines } \\ \text { Ausdruckes }\end{array}\right.$ nennt."

B: (2) "Was ist denn das?"

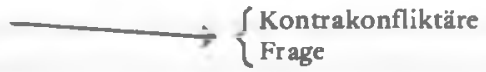

A : (3) "Eine kurze Lagebesprecbung, \} die manchmal aber docb länger dauert."

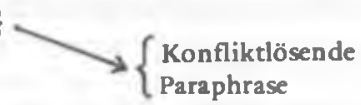

Kennt ein Adressat eine definitionsgemäße Verwendung eines Fachausdruckes $F$ nicht, dann kann dies u.a. heißen, daß er die Definition, 
relativ zu der der Äußerer $F$ verwendet hat, nicht kennt und daher nicht erschließen kann, was der Äußerer gesagt und gemeint hat. Kurz: Er weiß nicht, wovon die Rede war. - Dies bedeutet dann, daß der Äußerer und der Adressat bezüglich des fraglichen Fachausdruckes $F$ nicht über das gleiche Sprachvermögen verfügen, was zugleich heißt, daß sie über den gerade verhandelten Sachverhalt kein gemeinsames Fachwissen haben. Der Adressat weiß nicht, was der Fachausdruck $F$ definitionsgemäß zu wissen gibt; relativ zum Äußerer hat er eine individuelle Lücke im Fachausdruck-Inventar und damit eine Fachwissenslücke. ${ }^{39}$ -

Hat ein Äußerer einen Fachausdruck $F$ verwendet, den der Adressat nicht kennt, und hat dieser Adressat die kommunikative Absicht zu verstehen, dann ergeben sich für den Adressaten eine Reihe von Möglichkeiten, seine Verstehensabsicht zu verwirklichen. Diese Möglichkeiten können durch Bedingungen, die auf der Ebene der situationellen und/oder institutionellen Pragmatik zu formulieren sind - und die ich hier beiseite lassen muß mehr oder weniger stark eingeschränkt werden. Auf jeden Fall muß der Adressat dem Außerer sprachlich zu erkennen geben, daß er den fraglichen Fachausdruck $F$ nicht kennt und dies heißt immer auch, daß er eine Fachwissenslücke zu erkennen geben muß.

Der Adressat, der $F$ nicht kennt, hat zahlreiche Möglichkeiten, aufgetretene Sprachverstehenskonflikte zu beseitigen. Durch den Vollzug verschiedener kontrakonfliktärer Sprechhandlungen z.B. kann er ein metakommunikatives Interludium initiieren, das zur Behebung des akuten Konfliktes führen kann. Nachfolgend gebe ich, mehr oder weniger schematisiert, einige Möglichkeiten für konfliktbenennende Äußerungen an, die initiative, kontrakonfliktäre Sprechhandlungen ausdrücken.

(1) Fragen stellen:

Wie gebrauchen Sie $F$ ? Was heißt $F$ ? Was bedeutet $F$ ? Was ist ein $F$ ? Was meinen Sie denn mit $F$ ? Wie ist $F$ definiert? Können Sie mir erklären, was Sie unter $F$ verstehen?

Ein solcher Fall liegt im Beispiel Nr. 14 vor.

(2) Aufforderungen/Bitten äußern:

Sie müssen mir zunächst erläutern, was Sie unter $F$ verstehen! Würden Sie bitte mal näher erläutern, was Sie unter dem Begriff $F$ verstehen. Solche Aufforderungen finden sich in den Beispielen Nr. 17 und Nr. 24.

(3) Vorwürfe äußern:

Sie wissen doch genau, daß ich von Tagmemik nichts verstehe und mithin $F$ mir nicht geläufig ist. Als Vorwurf (mit anschließender Aufforderung) läßt sich das Beispiel Nr. 16 auffassen. 
Mußt Du eigentlich laufend $F$ s aus der Logik benutzen?

(4) Bedauern ausdrücken:

Es tut mir leid! Mit dem Terminus $F$ kann ich wenig anfangen.

(5) Fragend/vermutend Hypothesen zur möglichen Semantisierung von $F$ anbieten. Hierher gehören die Beispiele Nr. 7, Nr. 25 und Nr. 36, (2).

Je nachdem, von welcher Art der fragliche Fachausdruck $F$ ist, verläuft die auf den akuten Konflikt bezogene, eingebettete metakommunikative Handlungssequenz unterschiedlich. Vergleicht man z.B. wortsemantisch bedingte Sprachverstehenskonflikte in nicht-wissenschaftlichen Gesprächen - ein Fall liegt im Beispiel Nr. 36, (3) vor - mit solchen, die durch nicht-normierte Termini in wissenschaftlichen Gesprächen zustande kommen, kann man bestimmte Unterschiede feststellen. Nach meinen Beobachtungen gelingt in nicht-wissenschaftlichen Gesprächen die Konfliktlösung häufig bereits mit der- oder denjenigen Sprechhandlungen, die unmittelbar nach nur einem Sprecherwechsel auf die konfliktindizierenden Äußerungen folgen. Dies heißt auch: Der Anschluß an vorhandenes Wissen gelingt häufig in einem Gesprächsschritt. Dies gilt auch für solche Fachausdrücke, die nicht definiert sind, und die ich in der Typologie hinsichtlich ihrer Bedeutung als pragmatisch eingespielt gekennzeichnet habe. Ist $F$ jedoch ein zentraler Terminus eines theoretischen Konzeptes - wie etwa im Gesprächsausschnitt (1) im Beispiel Nr. 36 - d.h. ein Fachausdruck, der seinerseits nur hinreichend erklärt werden kann, indem andere Termini der Theorie zur Erklärung herangezogen werden müssen, dann führt dies häufig zu neuen Kommunikationskonflikten.

In einen konzentrierten Fachgespräch zwischen zwei Fachkollegen wird es eventuell gelingen, a uch in einem solchen Fall zum ursprünglichen $\mathrm{Ge}$ sprächsziel zurückzukehren, indem man von bestimmten gesprächssteuernden Verfahrensweisen Gebrauch macht, die z.B. in folgenden mehr oder weniger festen Redewendungen angedeutet sind: den Exkurs abbrecben, den roten Faden wieder aufnebmen, ein Problem ausklammern, einer Sache nicht weiter nachge.ben, zum Ausgangspunkt zurückkebren u.a. -

Sehr anders und zum Teil komplexer strukturiert sind solche semantisch bedingten Kommunikationskonflikte, die in der fachinternen Kommunikation durch die Verwendung solcher Fachausdrücke entstehen, die entweder mehrdeutig sind, d.h. im Fach mehrere, beispielsweise schulspezifische Gebrauchsweisen haben, oder im Verlauf der Fachgeschichte zahlreiche ähnliche Definitionen gefunden haben und durch solcherart disziplininterner Terminologieverschiebung einen mehr oder weniger instabilen Gebrauch haben, der sich in zahlreichen nuancenreichen, stets 
klärungsbedürftigen Verwendungen zeigt. M.E. sind das solche Fachausdrücke wie Langue, Kompetenz, Transfer, Intention, Hörversteben, Bedeutung, Wort, Wortklasse, Bebauptung, gelingen von Sprecbakten u.a. Während z.B. im Falle von Transformation eine typisch konfliktprophylaktische Wendung wie: Icb gebraucbe $F$, (hier also Transformation), im Sinne von Harris, ihre kommunikative Funktion unter bestimmten Bedingungen noch erfüllen kann, ist dies im Falle von Langue in der Wendung: Ich gebraucbe Langue im Sinne von Saussure kaum der Fall, was mit der Kommunikationsgeschichte des Ausdrucks Langue zusammenhängt.

Semantisch bedingte Kommunikationskonflikte, die durch Unkenntnis eines Fachausdruckes oder einer nicht geläufigen Verwendungsweise eines Fachausdruckes zustande kommen, treten nicht nur in der mündlichen Fachkommunikation, sondern auch in Fachlektüresituationen auf, z.B. öfters bei der Einarbeitung in ein neues Teilgebiet. Häufig reichen dann zum Erreichen des Textverstehens einerseits die sprachgebundenen Fachkenntnisse des Lesers nicht aus und andererseits auch nicht diejenigen Verfahren und indirekten Hilfen in wissenschaftlichen Texten, die eigens zur prophylaktischen Verstehenssicherung bzw. Lösung eventuell auftretender akuter Sprachverstehenskonflikte bei der Textlektüre vom Autor angewandt bzw. mitgegeben werden. Verfahren, die nicht ausschließlich aber stets auch der unmittelbaren Verstehenssicherung dienen, und die sich vor allem auf die Semantik von Fachausdrücken beziehen, sind z.B.: Explizite Festsetzungsdefinitionen oder Ketten von solchen, Abbildungen wie Diagramme etc., Vermeidung von nicht explizit gemachter terminologischer Synonymie und zahlreiche andere. Man darf - nebenbei bemerkt - solche Verfahren nicht z.B. mit korrekter Argumentation, stringenter Beweisführung und ähnlichem auf eine Ebene stellen. Eine korrekte Argumentation kann eben auch dann vom Leser nicht nachvollzogen werden, wenn sie mit Fachausdrücken operiert, die der Leser nicht kennt. Indirekte Hilfen für eventuelle Konflikte bei der Textlektüre, die durch die Semantik von Fachausdrücken entstehen, sind z.B.: Texttranszendierende Verweise auf eine Definition bei oder in X, expliziter Ausschluß von eventuell naheliegenden Semantisierungsmöglichkeiten für einen Fachausdruck, textinterne Verweise, Literaturverweise, Vorwortpassagen u.a. -

Trotz dieser und zahlreicher anderer Möglichkeiten, die einem Textautor zur Verfügung stehen, kommt es dennoch häufig vor, daß einem Fachtextleser die Semantisierung eines fraglichen Fachausdruckes nicht oder nur zu vage gelingt, so daß das weitere Textverstehen gefährdet ist. Es liegt dann ein kommunikativer Konflikt vor, und der Leser befindet sich in 
einer Fragesituation, in der er verschiedene Fragehandlungen ausführen kann. U.a. kann er zu einem Fachwörterbuch greifen. Mit diesem Griff nach dem Fachwörterbuch wird aus der Fragesituation eine Fachwörterbuch-Benutzungssituation. Eine solche Situation gebe ich im folgenden Beispiel wieder.

\section{BEISPIEL Nr. 37}

Ein Leser $\mathrm{L}$ liest einen fachwissenschaftlichen Text, in dem folgender Ausschnitt T vorkommt:

$\mathrm{T}$ : Die phonologisch determinierten Allomorphe (=F) machen erfahrungsgemäß im Fremdsprachenunterricht bestimmte Schwierigkeiten.

L stolpert über den Fachausdruck $F$.

L weiß zwar, was Allomorphe sind, und er weiß auch, was determiniert und was phonologiscb heißt. Was jedoch $F$ genau bedeutet bzw, was phonologisch determinierte Allomorphe genau sind, weiß er nicht. Die Semantisierung von $F$ gelingt immer nur ungefähr. Aus der Kenntnis der drei Wörter, aus denen $F$ besteht, weiß $L$ zwar, daß phonologisch determinierte Allomorphe solche sein müssen, die irgendwie phonologisch determiniert sind. Die Unterklasse derjenigen Allomorphe, die so bestimmt sind, ist jedoch für $\mathrm{L}$ leer, d.h. L kann $F$ nicht auf ein Element dieser Unterklasse beziehen.

L schlägt in einem Fachwörterbuch nach (Abraham: Terminologie zur neueren Linguistik). Dort findet er:

ALLOMORPH, phonologisch determinierte: Phonemisch verschiedene Allomorphe, deren Vorkommen von der phonologischen Umgebung abhängig ist: z.B. "bad-ete", "wett-et-e" [also nach Dental] gegenüber "lieb-t-e", "hack-te" [nach anderen als Dental]. Demnach entweder $e t$ oder $t$ als Präterialmorphem. nach Funkkolleg 3, 1971: 92.

Nach der Lektüre dieses Fachwörterbuchartikels ist der fachsemantisch bedingte Sprachverstehenskonflikt beseitigt und L's individuelle Wissenslücke geschlossen.

Damit dürfte wenigstens plausibel sein, daß Fachwörterbücher zwar nicht nur, aber doch auch als Bücher zu sehen sind, die gerade dazu gemacht werden, um Kommunikationskonflikte, insbesondere solche, die bei der Textlektüre entstehen, lösen zu helfen. Die je konsultierten Fachwörterbuchartikel werden bei ihrer Lektüre - und das ist der günstigste Fall zu kontrakonfliktären, konfliktlösenden Texten in Funktion. Sie können auch neue Kommunikationskonflikte schaffen. Erarbeitet man eine möglichst detaillierte Typologie von Fachwörterbuchbenutzungssituationen, dann ergibt sich dadurch m.E. die Möglichkeit, die Struktur von Fachwörterbuchartikeln relativ zu verschiedenen Klassen von Fach- 
lemmata besser zu gestalten als das m.E. bisher der Fall ist. 40

Nicht nur die Fachwörterbücher und die fachsprachliche Lexikographie ist im Zusammenhang mit der Verhinderung bzw. Beseitigung von akuten Kommunikationskonflikten zu sehen, sondern auch die verschiedenen Institutionen, die sich mit der Sprachnormierung, insbesondere der Terminologienormung befassen, wie z.B. das "Deutsche Institut für Normung". Man kann durchaus sagen, daß das oberste Ziel jeder Terminologiearbeit und der sich daran anschließenden Terminologienormung die prophylaktische Verhinderung von fachsprachlich bedingten Kommunikationskonflikten ist. Dabei gilt, daß diese Normierungshandlungen nicht um der Kommunikation willen vollzogen, sondern zweckrational begründet werden, d.h., sie geschehen im Dienste der Rationalisierung und Effektivierung volkswirtschaftlicher Prozesse. Mithin ist die Terminologienormung eine institutionalisierte Vorwegnahme derjenigen hermeneutischen A rbeit, die die Kommunikationspartner in der fachbezogenen Sprach kommunikation stets dann leisten müssen, wenn die jeweilige Verwendung von Fachausdrücken nicht eindeutig ist ${ }^{41}$, zu Sprachverstehenskonflikten auf der propositionalen Ebene geführt hat oder wenn der Gebrauch von Fachausdrücken zur Diskussion steht. Im allgemeinen will die Terminologienormung lediglich sicherstellen, daß die jeweiligen propositionalen Akte, die durch solche Äußerungen vollzogen werden, in denen normierte Fachausdrücke referierend und/oder prädizierend verwendet werden, wenigstens in den jeweiligen fachinternen, möglichst jedoch auch in interfachlichen Handlungsbereichen, für die beteiligten Kommunikationspartner eindeutig sind. Um ein einfaches Beispiel zu nennen: Es soll z.B. verhindert werden, daß A, der bei B eine Freispannsäge bestellt hat, von B eine Strecksäge geliefert bekommt, oder, daß B erst telephonisch oder schriftlich zurückfragen muß, welcher Typ von Handsäge denn eigentlich bestellt sei: Denn solche konfliktträchtige Kommunikation ist unwirtschaftlich und ineffektiv. - Insbesondere für den Bereich der technischen Fachsprache ist die Terminologienormung heute wohl unentbehrlich. Für den Bereich der Sprachwissenschaften, und zwar auch für die sog. angewandten Bereiche - mit Ausnahme vielleicht spezieller Programmierungssprachen - , ist Terminologienormung jedoch prinzipiell abzulehnen. Man kann zwar die Bezeichnung für 150 verschiedene Schraubenarten getrost normen. Den Schrauben tut das nicht weh, und die Benutzer der normierten Schraubenbezeichnungen sind nur dann betroffen, wenn die Normung den bereits eingespielten Sprachgebrauch zu wenig berücksichtigt hat. Sozialwissenschaftliche Fachausdrücke aber zusammen mit ihren Definitionen konstituieren in vielen Fällen erst den wissenschaftlichen Gegenstand. Normung hieße hier auf möglichen Erkenntnisgewinn oder -zuwachs weitgehend verzichten, da jede Reflexion auf den fachspezifischen Sprach- 
gebrauch und damit auch das Nachdenken über manche wissenschaftlichen Gegenstande letztlich mit einem Hinweis auf die Norm enden muß. Eine 'gewisse Erleichterung, die man vielleicht in bestimmten angewandten Bereichen, z.B. im Grammatikunterricht, zunächst bei der Erkenntnisvermittlung und der Vermittlung von Fachwissen möglicherweise hätte, würde nach kurzer Zeit zu unüberbrückbaren Gräben, nämlich zu fachsprachlichen Barrieren und damit Wissensunterschieden zwischen denen führen, die stupide normgerechte Erkenntnis vermitteln, und denen, die, z.B. im Bereich der Forschung, sich an die Normierungen nicht halten. Terminologienormung ist in der Sprachwissenschaft kein brauchbares Mittel, um fachsprachlich bedingte Kommunikationskonflikte von vornherein zu verhindern. -

Hier scheint mir nun auch der Ort zu sein, um einige Bemerkungen zur Einschätzung von fachsprachlich bedingten Kommunikationskonflikten im fachinternen Handlungsbereich einzuflechten, und ich bitte, das einschränkende Prädikat fachintern ausdrücklich zu beachten. Es ist keineswegs so, daß Kommunikationskonflikte lediglich als Ereignisse zu gelten haben, die es ständig ängstlich zu verhindern gilt. Mit der Art mancher sozialwissenscha ftlicher Gegenstände, ihrem so und so Gegebensein durch einen gerade so und so definierten Fachausdruck hängt es zusammen, daß Kommunikationskonflikte, insbesondere solche, die akut werden, häufig den Anstoß zu weiterem Nachdenken, zu fruchtbaren Diskussionen und zur Erkenntniserweiterung sind. Dies gilt allerdings nur unter der Voraussetzung, daß die pragmatischen Bedingungen derart sind, daß z.B. Wissenslücken sanktionslos gezeigt werden können, Metakommunikation nicht mehr oder weniger tabuisiert ist, die soziale Schutzfunktion der Kommunikationskonflikte nicht zu häufig in Anspruch genommen werden muß und daher lieber latente Kommunikationskonflikte in Kauf genommen werden, weil zu fürchten ist, daß aus akuten kommunikativen Konflikten Meinungskonflikte entstehen können, die man - schon darin eingespielt lieber vertuscht bzw. die einer der beteiligten Kommunikationspartner und hier wäre der schöne Ausdruck Kommunikationspartner ein Euphemismus und besser durch Kommunikationsunterworfener zu ersetzen meiden muß, weil er sich solche möglichen Meinungskonflikte aufgrund seiner Stellung im Wissenschaftsbereich oder wegen seiner Beziehungen zu den geschätzten Partnern deswegen nicht leisten kann, weil eventuelle Sanktionen kaum einzuschätzen sind. - Hier wäre nun der Punkt, wo man zu denjenigen Kommunikationskonflikten übergehen müßte, die situativ bzw. institutionell bedingt und empirisch-pragmatisch zu analysieren wären. Dabei käme es nicht nur darauf an, solche Konflikte zu studieren, die z.B. durch Verletzung des kommunikativen Kooperationsprinzips und der sog. Konversationsmaximen 42 zustande kommen, 
sondern auch solche, die mit den institutionellen Bedingungen von wissenschaftlicher Tätigkeit überhaupt zu tun haben. Dabei wäre wohl davon auszugehen, das wissenschaftliches Sprechen - insbesondere auch auf öffentlichen Großtagungen - etwa Sprechen nach dem Muster 'einen wissenschaftlichen Vortrag halten' oder nach dem Muster 'einen Diskussionsbeitrag leisten' meistens in dem guten Glauben geschieht, vornehmlich auf den gerade verhandelten wissenschaftlichen Gegenstand bezogen zu sein, selten aber nur auf diesen allein bezogen ist!

Daher sind auch Tagungen nicht nur Stätten der wissenschaftlichen Kooperation, des sachlichen Informationsaustausches, der Erkenntnisvermittlung und -gewinnung und der kooperativen Wahrheitsfindung, sondern Tagungen sind stets auch Jahrmärkte der wissenschaftlichen Eitelkeit, was besondere Typen von Kommunikationskonflikten zu Folge haben kann, die auf der Beziehungsebene ${ }^{43}$ von $\mathrm{F}$ achkommunikation liegen.

\section{Schlußbemerkung}

Ich habe mich bemüht, das gegebene Thema - entgegen dem Trend, der in der Literatur zur Erforschung von Kommunikationskonflikten zu beobachten ist - in informeller Weise zu behandeln. Ich hoffe daher, daß meine Ausführungen so waren, daß möglichst wenig Textpartien so verstanden worden sind wie folgendes Zitat aus der Züricher Zeitung vermutlich verstanden werden wird: "Das Kreuzworträtsel, das in der Nummer von heute erscheinen sollte, stand stattdessen in der von gestern, zusammen mit der Lösung des Rätsels, das gestern hätte erscheinen sollen. Das Rätsel, das für gestern vorgesehen war, steht daher in der Nummer von heute, zugleich mit der Lösung des Rätsels vom Montag. Das Rätsel für heute und die Lösung, die gestern hätte erscheinen sollen, bringen wir morgen."

\section{Anmerkungen}

1 Der Wortlaut des Vortrages wurde in dieser schriftlichen Fassung weitgehend beibehalten. Anm. wurden ergänzt, das umfangreiche Handout nur teilweise eingearbeitet; dadurch wurden Umformulierungen und Textergänzungen notwendig.

2 Diese "These" ist das Ergebnis meiner Einschätzung der neueren Fachsprachenforschung. Zwar ist neuerdings deutlich geworden, daß Fachsprachenforschung nicht vornehmlich aus der Erforschung von Fachwortschätzen, spezifischen Wortbildungsmustern und -mitteln, syntaktischen Besonderheiten in bestimmten Textsorten sowie statistischen Untersuchungen bestehen kann, vgl. u.a. Möhn 1977, 67 ff; der Forschungsbericht von Bergmann/Zapf 1965 zeigt jedoch ex negativo, daß z.B. die Erforschung des 
$\mathrm{G} \mathrm{e} \mathrm{b} \mathrm{r} \mathrm{a} \mathrm{u} \mathrm{c} \mathrm{h} \mathrm{s} \mathrm{von} \mathrm{Fachsprachen} \mathrm{im} \mathrm{Industriebetrieb} \mathrm{noch} \mathrm{gänzlich} \mathrm{in}$ den Anfängen steckt. Die Arbeit von H. Schönfeld u. J. Donath: Sprache im sozialistischen Industr iegebiet. Untersuchungen zum Wortschatz bei sozialen Gruppen. Berlin-Ost 1978 (Sprache und Gesellschaft) war mir leider nicht zugänglich.

3 Es gibt relativ zahlreiche Untersuchungen zur Struktur und Entwicklung von wissenschaftlichen Fachsprachen. Davon kann man sich durch einen Blick in die einschlägigen Bibliographien (z.B. Barth 1971) oder die Einführungs- bzw. Übersichtsbücher (z. B. Hoffmann 1976, Fluck 1976, Drozd/ Seibicke 1973) überzeugen. - Untersuchungen zum G e b r a u $\mathrm{ch}$ von wissenschaftichen Fachsprachen z.B. in Kolloquien, Projektgruppen, im Rezensionswesen, in Zeitschriftendiskussionen, in öffentlichen wissenschaftlichen Tagungen, im Gutachterwesen, in Projektanträgen usw. liegen kaum vor. Es gibe lediglich einige Arbeiten zum Argumentationsstil, 2.B. Geier/ Keseling/Nehrkorn/Schmitz 1977. - In verschiedenen Arbeiten zur Sprache in der Jurisprudenz finden sich ebenfalls Hinweise zum Gebrauch der juristischen Fachsprache. Diese Arbeiten sind zum Teil erschließbar über folgende Materialsammlung: Ladnar/von Plottnitz (Hrsg.) 1976.

4 Daher nehme ich die Beispiele überwiegend aus der linguistischen Fachsprache bzw. aus Diskussionen zwischen Sprachwissenschaftlern. Zu dem in (3) bezogenen Standpunkt vgl. Ungeheuer im Vorwort zu Richter/Weidmann 1975.

Der Ausdruck Zwecksprache ist in der Terminologieforschung gebräuchlich; vgl. z.B. Wüster 1970.

Bekannte Bemühungen in dieser Richtung können allenfalls Teilerfolge verbuchen und sind selbst in verschiedenen Hinsichten konfliktträchtig. Dies gilt z.B. für Konstruktion von speziellen Sprachen und für die Terminologienormung. Auch besonders explizites wissenschaf tiches Sprechen kann unter Umständen gerade zur Kommunikationsverweigerung führen.

7 Vgl. z. B. Richter/Weidmann 1975 und Backhausen o.J.

8 Zum Ausdruck Definitionskette vgl. Savigny 1970 und Wiegand 1978.

9 Zu nominalen Festsetzungsdefinitionen vgl. Wiegand 1978 und die dort verzeichnete Literatur.

10 Zur Terminologisierung vgl. Drozd/Seibicke 1973, $147 \mathrm{ff}$.

11 Vgl. dazu Albert 1975.

12 Mit Keller 1977 (und der dort zu dieser Frage angegebenen Literatur) gehe ich davon aus, daß Verstehen nicht ein Prozeß, sondern das Ergebnis eines Prozesses ist.

13 Zu dieser Diskussion vgl. u.a. Wunderlich 1976, $110 \mathrm{ff}$. und die dort verzeichnete Literatur und Wunderlich 1972, 22 ff. sowie Mass 1972, 296 ff.

14 Zum Ausdruck Wissensraum vgl. Rehbein 1977, $35 \mathrm{ff}$.

15 Zum Ausdruck Handlungsraum vgl. Rehbein 1977, $12 \mathrm{ff}$.

16 Quasi-empiriscb können Untersuchungen heißen, die mit Beispielen arbeiten, die der sprachkompetente Untersuchende selbst regelgerecht bildet. Vgl. dazu u.a. Rehbein 1977, 4, 7, 10. 
Ahnliche methodische Ansicht bei Rehbein 1977, 3 ff. - Vgl. dazu auch meine Bemerkungen zur hermeneutischen Position im Abschnitt 4.

18 Hier wären dann diejenigen Verwendungsweisen von besonderem Interesse, die sich auf das Verstehen von sprachlichen Außerungen beziehen. Eine semantische Untersuchung zum Gebrauch des Ausdruckes versteben in nichtwissenschaftlichen Texten ist mir nicht bekannt. Nach den mir vorliegenden Belegen könnte eine solche Untersuchung wahrscheinlich erstens zeigen, daß im nichtwissenschaftlichen Gebrauch feinere semantische Unterscheidungen und Nuancierungen vorliegen als im wissenschaftlichen und philosophischen Sprachgebrauch, und zweitens, daß insbesondere prominente Hermeneuten die vielfache Polysemie dieses Ausdruckes nicht hinreichend berücksichtigt haben.

19 Vgl. dazu Jäger 1978 sowie meine Bemerkungen zur hermeneutischen Position in Abschnitt 4.

20 Zu den klassifikatorischen Begriffen vgl. v. Kutschera 1972, 16 ff.; ich meine allerdings, daß es zu weniger unangenehmen Konsequenzen führt, wenn man von klassifikatorischen Ausdrücken bzw. Fachausdrücken spricht; es sei denn, man gibt nachvollziehbare Regeln für den Gebrauch des Ausdruckes Begriff an. Vgl. dazu Wiegand 1978 und die dort verzeichnete Literatur.

21 Die Grenze zwischen Kommunikationskonflikten und Meinungskonflikten z.B. ist derzeit m.E. keineswegs klar abzustecken.

$22 \mathrm{Zu}$ bestimmten Typen von Kommunikationskonflikten gehören bestimmte Typen von Konfliktindikatoren. Daher könnte man die Beispiele Nr. 2 bis Nr. 34 systematisch ordnen, was ich hier jedoch unterlassen will, weil dazu erheblich detailliertere Ausführungen notwendig wären.

23 Meine Belege für diese Ausdrücke stammen aus der "Zeit", dem "Zeitmagazin", dem "Spiegel" sowie aus der Dudenkartei. Auch habe ich mehrere einsprachige Wörterbücher und Fachwörterbücher zum Vergleich eingesehen.

24 Zum Ausdruck Interaktionsraum vgl. Rehbein 1977, 12, 21 ff. u. $186 \mathrm{ff}$.

25 Ich verweise vor allem auf Ungeheuer 1972 und 1977; den hier entwickelten Ansichten zur Kommunikation stimme ich in den meisten Punkten zu, wenn ich z. Teil auch andere Ausdrücke verwende. Dafür habe ich Gründe, die ich hier nicht en detail darlegen kann; sie hängen jedoch damit zusammen, daß ich den Gebrauch z.B. der Ausdrücke: Versteben, Spracbversteben, Verständnis, Verständigung u.a. gerne im Anschluß an ihren nichtwissenschaftlichen Gebrauch in anderer Weise auseinanderhalten möchte.

26 Im Folgenden werde ich einige Fachausdrücke, die im Rahmen der sog. Gesprächsanalyse üblich sind, verwenden. Es handele sich z.B. um die Ausdrücke Gespräch, Gespräcbsverlauf, Gespräcbseröffnung, Gesprächsbereitschaft, Gespräcbszustand, Gespräcbsziel, Gesprächsscbrittsequenz und einige andere. Diese sind hier so verwendet, daß sie auch ohne die Bereitstellung möglicher Definitionen ausreichend verstanden werden können. Wer zu einem intensiveren Verständnis vordringen möchte, kann sich folgender Arbeiten und der dort verzeichneten Literatur mit Nutzen bedienen: Berens et al. 1976; Schank/Schoenthal 1976; Kallmeyer/Schütze 1976; Wunderlich 1976, 293 - 395. Henne 1977; Henne/Rehbock 1978. 
Den Ausdruck Spracbversteben möchte ich hier nicht eingehender erläutern, nur ergänzend auf folgendes hinweisen. In einem Gespräch liegt vollständiges Sprachverstehen erst dann vor, wenn ein Angesprochener wenigstens Hörverstehen, propositionales Verstehen, illokutionäres Verstehen, kollokutionäres Verstehen und gesprächsstrategisches Verstehen erreicht hat. Entsprechend dieser analytischen Dekomposition von Spracbversteben lassen sich auch verschiedene Typen von Sprachverstehenskonflikten angeben. - Wenn ein A den B und ein B den A sprachlich verstanden hat, heißt dies aber noch keineswegs, daß z.B. am Ende einer Gesprächsschrittsequenz Verständigung zwischen $\mathrm{A}$ und $\mathrm{B}$ erreicht ist. Schon im nicht-wissenschaftlichen Sprechen macht man hier erheblich feinere Unterschiede. So sagt man z.B. Hinz und Kunz konnten sich nicht verständigen oder ... Eine Verständigung zwischen Ludwig und Erich ist nicht zustande gekommen. Wer diese Sätze äußert, meint aber nicht notwendigerweise, daß sich die beiden sprachlich nicht verstanden haben. - Zum Ausdruck kollokutionär bzw. kollokutionärer Akt vgl. Keller 1977a.

28 Dies heißt, daß der Adressat vollständiges Sprachverstehen (vgl. Anm. 27) nicht erreichen konnte. Das Prädikat erfolgreicb sein (auf der ersten Ebene) verwende ich im Anschluß an Wunderlich 1976, 115 ff. Im Unterschied zu Wunderlich bin ich jedoch der Meinung, daß zum vollständigen Sprachverstehen mehr gehört als das Erkennen des Adressaten, daß der Sprecher eine bestimmte (propositionale) Einstellung ausdrückt.

29 Dazu vgl. Ungeheuer 1972.

30 Den Ausdruck kontrakonfliktär übernehme ich von Backhausen o.J. bzw. von Ungeheuer in Richter/Weidmann 1975.

31 Zur Verwendung des Ausdruckes Interaktionsbedingung vgl. Wunderlich $1976,89 \mathrm{ff}$.

32 Zur Unterscheidung von reaktiven und initiativen Sprechakten vgl. Wunderlich $1976,76 \mathrm{ff}$.

33 Zu diesem Typ von Metakommunikation vgl. Wiegand 1978a; dort ausfuhrliche Literatur zur Metakommunikation.

34 Für alle hier aufgelisteten Typen finden sich in den Beispielen Nr. 2 bis Nr. 34 Konfliktindikatoren.

$35 \mathrm{VgL}$. Dubach/von Rechenberg 1977.

36 Das hängt z.B. damit zusammen, daß bei der Verwendung von Fac'ıausdrücken keineswegs nur deren Referenz- oder Prädiakionsfunktion eine kommunikative Rolle spielt. Wer einen bestimmten Fachausdruck $F$ verwendet, gibt u.U. damit bereits implizit zu erkennen, welche wissenschaftliche Position er vertritt, und bereits dies kann eine Bewertung des Gesprächspartners bedeuten, die u.U. den weiteren Gesprächsverlauf beeinflussen kann.

37 Die mir bekannten Typologien von Fachausdrücken sind $m$.E. ziemlich heterogen, da sie meistens ganz verschiedene Kriterien verwenden. Vgl. z.B. Schmidt 1969, 20; Fluck 1976, 47 ff. Die Diskussion bei Hoffmann 1976, 259 ist zwar sehr breit angelegt, aber auch hier wird nicht ausreichend berücksichtigt, daß man nicht zugleich alle Eigenschaften von Fachausdrücken berücksichtigen kann, wenn man Klassifizierungen anstrebt. 
39 Der Zusammenhang von Fachwissen und der Beherrschung einer Fachsprache bzw. der Kenntnis von Fachausdrücken ist sicherlich kompliziert; mir kommt es hier nur darauf an, daß er besteht.

40 Mit diesen Fragen habe ich mich - anhand einsprachiger Wörterbücher in Wiegand 1977 befaßt.

41 Vgl. dazu auch Bausch 1976.

42 Vgl. Grice 1975.

43 Die sog. Beziehungsebene von Kommunikation ist erstens nicht metakommunikativ und zweitens läßt sie sich von der Inhaltsebene keineswegs strikt trennen. Vgl. z.B. die Diskussion bei Boettcher/Sitta 1978, 42 ff. und Wiegand 1978a.

\section{Lireratur}

Albert, Hans 1975: Transzendentale Träumereien. Karl-Otto Apels Sprachspiele und se in hermeneutischer Gott. Hamburg 1975 (Standpunkt. Analysen. Dokumente. Pamphlete).

Backhausen, Wilhelm J. o.J.: Grundzïge eines Kommunikatormodells als STSystem. Hamburg o.J. (= IPK-Forschungsberichte 41).

Barth, Erhard 1971: Fachsprache. Eine Bibliographie. In: Germanistische Linguistik 1971. H. 3, 205 - 363.

Bausch, Karl-Heinz 1976. : Fach- und Gemeinsprache als kommunikationssoziologisches Problem. In : Karl-Heinz Bausch/Wolfgang H.U. Schewe/HeinzRudi Spiegel: Fachsprachen. Terminologie. Struktur. Normung. Berlin. Köln 1976 (= Normungskunde Heft 4), 124 - 136.

Bergmann, J.W. Zapf 1965: Kommunikation im Industriebetrieb. Ein Bericht über den Stand der deutschen Forschung. Stuttgart 1965.

Berens, Franz-Josef/Karl-Heinz Jäger/Gerd Schank/Johannes Schwitalla 1976: Projekt Dialogstrukturen. Ein Arbeitsbericht. Mit einer Einleitung von Hugo Steger. Mün chen 1976 (= Heutiges Deutsch I, Bd. 12).

Boettcher, Wolfgang/Horst Sitta 1978: Der andere Grammatikunterricht. München. Wien. Baltimore 1978.

Drozd, Lubomir/Wilfried Seibicke 1973: Deutsche Fach- und Wissenschaftssprache. Bestandsaufnahme - Theorie - Geschichte. Wiesbaden 1973.

Dubach, U.C./K.-M. von Rechenberg 1977: Krankheitsverständnis und Patienten Arzt - Beziehung in der Ambulanz. In: Deutsche Medizinische Wochenschrift $35,1977,1239-1244$.

Fluck, Hans-Rüdiger 1976: Fachsprachen. Einführung und Bibliographie. München 1976 (= Uni-Taschenbücher 483). 
Geier, Manfred/Gisbert Keseling / Marianne Nehrkorn / Ulrich Schmitz 1977: Zum Beispiel: Argumentieren. Ein Beitrag zum Verhältnis von synchroner, ontogenetischer und historischer Rekonstruktion. In: Klaus Baumgärtner (Hrsg.): Sprachliches Handeln [...]. Heidelberg 1977 (= medium literatur 7), $69-108$.

Grice, H. Paul 1975: Logic and conversation. In: P. Cole / J. Morgan (Hrsg.): Speech acts. New York 1975 (= Syntax and Semantics 3).

Hoffmann, Lothar 1976: Kommunikationsmittel Fachsprache. Eine Einführung. Berlin 1976 (= Sammlung Akademie-Verlag 44. Sprache).

Henne, Helmut 1977: Gesprächsanalyse - Aspekte einer pragmatischen Sprachwissenschaft. In: Dirk Wegner (Hrsg.): Gesprächsanalysen [...] Hamburg 1977 (= IPK-Forschungsberichte, Reihe I, Bd. 65), 67-92.

Henne, Helmut / Helmut Rehbock 1978: Einführung in die Gesprächsanalyse. Berlin. New York (Göschen) [erscheint].

Jäger, Ludwig 1978: Zu einer hermeneutischen Begründung der Sprachtheorie. Historisch-systematische Skizze. In: Germanistische Linguistik 1977 [erscheint].

Kallmeyer, Wemer / Fritz Schütze 1976: Konversationsanalyse. In: Studium Linguistik 1. 1976, 1 - 28.

Keller, Rudi 1977: Verstehen wir, was ein Sprecher meint, oder was ein Ausdruck bedeutet? Zu einer Hermeneutik des Handelns. In: Klaus Baumgärtner (Hrsg.): Sprachliches Handeln [...] . Heidelberg 1977 (= medium literatur 7) 1 - 27.

- - 1977a: Kollokutionäre Akte. In: Germanistische Linguistik 1977. H. 1 - 2, $3-45$.

Kutschera, Franz von 1972: Wissenschaftstheorie I. Grundzüge der allgemeinen Methodologie der empirischen Wissenschaften. München 1972 (= UniTaschenbücher 100).

Ladnar, Ulrike / Cornelia von Plottnitz 1976: Fachsprache der Justiz. Ein Arbeitsbuch für den Deutschunterricht und die Gemeinschaftskunde auf der Oberstufe (Kollegstufe) Frankfurt/Main. Berlin. München 1976 (Kommunikation/Sprache. Materialien für den Kurs- und Projektunterricht).

Maas, Utz 1972: Grammatik und Handlungstheorie. In: Utz Maas/Dieter Wunderlich, Pragmatik und sprachliches Handeln. Mit einer Kritik am Funkkolleg 'Sprache'. Frankfurt 1972 (= Athenäum-Skripten Linguistik 2) 189 - 276.

Möhn, Dieter 1977: Ziele und Ergebnisse der Fachsprachenforschung und der Terminologiearbeit. In: Muttersprache 87, 1977, 67 - 76.

Rehbein, Jochen 1977: Komplexes Handeln. Elemente zur Handlungstheorie der Sprache. Stuttgart 1977.

Richter, Helmut / Fred Weidmann 1975: Semantisch bedingte Kommunikationskonflikte bei Gleichsprachigen. Mit einem Vorwort von Gerold Ungeheuer. 2. durchgesehene Aufl. Hamburg 1975 (= IKP-Forschungsberichte 17).

Savigny, Eike von 1970: Grundkurs im wissenschaftlichen Definieren. Übungen zum Selbststudium. München 1970 (= dtv. WR 4062). 
Schank, Gerd/Gisela Schoenthal 1976: Gesprochene Sprache. Eine Einführung in Forschungsansätze und Analysemethoden. Tübingen 1976 (= Germanistische Arbeitshefte 18).

Schmidt, Wilhelm 1969: Charakter und gesellschaftliche Bedeutung der Fachsprachen. In: Sprachpflege 18. 1969, $10-21$.

Terminologie zur neueren Linguistik. Zusammengestellt von Werner Abraham unter Mitwirkung von R. Elema, R. Griesen, A.P. ten Cate und J. Kok. Tübingen 1974 (= Germanistische Arbeitshefte, Ergär,zungsreihe 1).

Ungeheuer, Gerold 1972: Was heißt "Verständigung durch Sprechen"? In: Gesprochene Sprache. Jahrbuch 1972 des Instituts für deutsche Sprache. Disseldorf 1974. (= Sprache der Gegenwart 26), 7 - 38.

- - 1977: Gesprächsanalyse und ihre kommunikationstheoretischen Voraussetzungen. In: Dirk Wegner (Hrsg.): Gesprächsanalysen. Vorträge, gehalten anläßlich des 5. Kolloquiums des Instituts für Kommunikationsforschung und Phonetik, Bonn, 14. - 16. Oktober 1976. Hamburg 1977 (= IKP-Forschungsberichte, Reihe I, Bd. 65), 27 - 65.

Wiegand, Herbert Emst 1977: Nachdenken über Wörterbücher. Aktuelle Probleme. In: Günther Drosdowski, Helmut Henne, Herbert E. Wiegand: Nachdenken über Wörterbücher. Mannheim. Wien. Zürich 1977, 51 - 102.

- 1978: Definition und Terminologienormung. Kritik und Vorschläge. [erscheint in Festschrift Wüster].

- - 1978a: Bemerkungen zur Bestimmung metakommunikativer Sprechakte. [erscheint in: Lunder Germanistische Forschungen].

Wüster, Eugen 1970: Intemationale Sprachnormung in der Technik besonders in der Elektrotechnik (Die nationale Sprachnormung und ihre Verallgemeinerung). 3. abermals erg. Aufl. Bonn 1970 (= Sprachforum. Beiheft Nr. 2).

Wunderlich, Dieter 1972: Zur Konventionalität von Sprechhandlungen. In: Dieter Wunderlich (Hrsg.): Linguistische Pragmatik. Frankfurt 1972 (= Schwerpunkte. Linguistik und Kommunikationswissenschaft 12), 11 - 58.

_ - 1976: Studien zur Sprechakttheorie. Frankfurt 1976 (suhrkamp taschenbuch wissenschaft 172). 